\title{
The design of transnational public good mechanisms for developing countries
}

\author{
Jean-Jacques Laffont ${ }^{\mathrm{a}, \mathrm{b}}$, David Martimort ${ }^{\mathrm{c}, \mathrm{d}, *}$ \\ ${ }^{\text {a } U n i v e r s i t e ́ ~ d e ~ T o u l o u s e, ~(I D E I, ~ G R E M A Q, ~ A R Q A D E), ~} 1$ Place Anatole, Toulouse Cedex 31042, France \\ ${ }^{\mathrm{b}}$ University of Southern California, Los Angeles, CA, USA \\ ${ }^{\mathrm{c}}$ Université de Toulouse, (IDEI, GREMAQ), France \\ ${ }^{\mathrm{d}}$ Institut Universitaire de France, France
}

Received 20 January 2003; received in revised form 26 February 2004; accepted 9 March 2004

Available online 1 June 2004

\begin{abstract}
We analyze the design of incentive mechanisms for the provision of transnational public goods under asymmetric information. Transnational public goods are infrastructures that no single country can afford to build for itself. We show that the external constraints imposed by this mechanism may affect consumption, pricing and the true redistributive concerns of local governments. We characterize the corresponding distortions. We also discuss the impact of the preferences for redistribution of the international agency in charge of designing the mechanism and the role of its ability to enforce that mechanism.

(C) 2004 Elsevier B.V. All rights reserved.
\end{abstract}

JEL classification: H41; D82

Keywords: Transnational public goods; Incentive mechanisms

\section{Introduction}

It is well recognized by now, both among practitioners and scholars, that proper infrastructures are key to economic development. Several empirical studies illustrate the impact of infrastructures on economic growth. ${ }^{1}$ A $1 \%$ increase in the stock of infra-

* Corresponding author. Université de Toulouse, (IDEI, GREMAQ), 1 Place Anatole, Toulouse Cedex 31042, France. Tel.: +33-61-12-86-38; fax: +33-61-12-86-37.

E-mail address: boe@cict.fr (D. Martimort).

1 See for the case of Latin America, Calderon et al. (2002) and Calderon and Serven (2002) among others. 
structures can increase GDP by up to $0.20 \%$. Despite this importance, some lowdeveloped countries suffer significant shortages in accessing to crucial infrastructures. For instance, the stock and quality levels of infrastructures in Latin America and Caribbean countries has lost significant ground relative to East Asia and OECD countries. To illustrate that point, Calderon and Serven (2002) show that, from 1980 to 1997, the Latin America infrastructure gap relative to East Asia grew by $40 \%$ for roads, $70 \%$ for telecommunications and nearly $90 \%$ for power generation. These authors argue that this widening infrastructure gap can account for nearly $25 \%$ of the Latin America's GDP output gap relative to the East Asian economies over the 1980-2000 period.

In response to this issue, and given the scarcity of public funds in LDCs, ${ }^{2}$ most developing countries have turned to the foreign private sector for financing and operating infrastructures. However, a number of difficulties have emerged from this strategy. First, some countries have failed to attract foreign investments. Second, even those who succeeded have sometimes faced a high rate of renegotiation for these contracts. ${ }^{3}$ Initiated by governments or concessionnaries, renegotiation has often created public opposition to what is sometimes presented, for water concessions in particular, as a loss of sovereignty.

Whatever the strength of the motivations behind these public positions, alternative ways of financing infrastructures should be looked for. How to reconcile the need for more investment in public infrastructures and the aspiration of LDCs for a close control of their public services is a major political question today.

Cooperation among small LDCs, such as those of Central America or of the Mediteranean Rim, might be a potential solution. Sometimes the least-cost approach to improving the supply of infrastructure services requires cross-country integration of networks or shared access to a common resource. This is certainly the case for infrastructure projects that arise out of the growth of commerce and trade. Examples include road and rail networks, power grids ${ }^{4}$ and telecommunication networks. This is also the case for projects which are less commercially driven such that investments in environmental protection and the management of shared resources such as water. ${ }^{5}$ This paper develops a theoretical framework to explore the allocative and distributive consequences of those transnational coordinations.

Infrastructures often entail fixed costs which are so large that no single country can afford to build the infrastructure alone. Those fixed costs must be shared by several

2 A recent report by the World Bank mentioned "When times are hard, capital spending on infrastructure is the first item to go... Despite the long-term economics costs of slashing infrastructure spending, governments find it less politically costly than reducing public employment or wages." World Development Report (1994, p. 19).

3 See Guasch et al. (2002).

4 A typical example of such a joint project is the Itaipu hydroelectricity power plant on the Brazil-Paraguay border. To give an idea of the scale of such a project, that dam corresponds to $25 \%$ of energy supply in Brasil and $78 \%$ in Paraguay.

5 Both kinds of transnational projects have recently been successfully implemented. Power links are already in place between Jordan and Syria, and between Syria and Lebanon. Plans have been developed to include Israel, the West Bank and Gaza but also other SubSaharan countries. An example of the second kind is given by the Marine Pollution Management Project developed by southwestern Mediterranean countries to enhance the capacity of countries like Algeria, Morocco, Tunisia, Lybia and Egypt to deal with marine pollution. 
countries. An infrastructure can thus be viewed as a public good for which some financing mechanism must be agreed upon by partners involved in the project. This perspective raises of course the ugly head of the free-rider problem which arises when an efficient Coasian bargaining fails. Such inefficiencies are particularly relevant in contexts plagued by informational asymmetries.

A general lesson from the literature on public good mechanisms developed over the last 30 years is that, under asymmetric information, the optimal mechanism may require some deviations away from the first-best. In our context, a country may pretend having a low willingness to pay for the public good to minimize its own contribution and let partners bear the bulk of investment. To curb those incentives, the project should be sometimes given up even though it would have been optimal to build it under complete information.

This important insight has been so far derived by looking at the provision of a public good for individual agents, not for countries. Countries can only be reduced to individual agents under the very restrictive assumption that all inhabitants are the same. Agents are in fact heterogeneous and may differ with respect to their wealth or their willingness to pay for the public good. Individual preferences are only aggregated through some political process. Politics determines the weights of the various types of agents in the objective functions of the governments involved in bargaining over the collective decision to build or not the common infrastructure.

In this paper, we argue that the most convenient framework to fully assess the allocative and distributive consequences of transnational public good mechanisms should make explicit this distinction between countries and individuals. The theoretical framework should account for two distinct layers of contracting and allow for a nested information structure. The first layer concerns the countries (or more precisely their governments) involved in bargaining. Countries are privately informed on the aggregate expected welfare they get from building the infrastructure and tend to minimize their respective contributions. The second layer deals with the fact that, within each country, agents have private information on the individual benefits they derive from the infrastructure. Optimal pricing of the service is then constrained by this added asymmetric information. The redistributive concerns of the governments affect pricing and may create a wedge between price and marginal cost to redistribute wealth across various groups.

With transnational projects, each country is externally constrained by the rules of the mechanism for collective choice. A collective solution to the bargaining problem, maybe designed under the aegis of an international agency (thereafter IA), defines each country's financial contribution to the common project and, whether it should be built or not. Different degrees of enforcement of these transnational mechanisms impose various external constraints on the redistributive concerns at the local level. The prices charged to consumers for using the infrastructure may then result from a compromise between these external constraints and the redistributive concerns of the governments. As a result, there is little hope to see transnational infrastructures being priced as simple "local public goods" which would have been self-financed by each country alone. To make the international negotiation easier, pricing policies in the different countries involved may be deeply intertwined. This paper precisely analyzes these distributive and allocative issues.

To set up the stage, we assume that an IA is in charge of raising contributions from two countries interested in building a transnational infrastructure and proposes a 
mechanism to be played by those countries. That mechanism determines the countries' contributions and the probability of building or not the infrastructure. Countries are made of heterogeneous agents with different preferences for the public good. At the aggregate level, countries may differ with respect to their preferences for redistribution and have private information on those preferences. Different levels of enforcement of the mechanism are analyzed.

In the most comprehensive contracting environment, the IA can suggest the prices charged to consumers and acceptance of the mechanism by each country is mandatory. The optimal mechanism which maximizes the sum of expected welfares in both countries selects the efficient ${ }^{6}$ decision to build or not the infrastructure and induces pricing schemes in each country which depend only on its own redistributive concerns.

The drawback of such an efficient mechanism is that incentive compatibility at the countries' level might be obtained by leaving the countries the most eager to redistribute with a negative level of expected welfare. When the possibility of opting out of the mechanism is taken into account, a country's incentives to report truthfully its preferences for redistribution may conflict with the exercise of those sovereign rights. The contribution to the collective project of a country that is eager to redistribute must be reduced. The project can no longer be performed as often as if the countries' preferences were common knowledge. Under weak conditions, pricing schemes in both countries are now intertwined. Asymmetric information between countries exacerbate redistributive concerns of the local governments and reinforce the conflict due to asymmetric information within the countries.

Contrary to standard public good problems where pricing and decision to build or not the infrastructure are jointly determined, the nested structure of our model suggests that the IA may not have the full control of the prices charged within each country. Instead, pricing may be still decided by governments if they cannot commit to relinquish these rights to the IA. The only remaining tool available to the IA for screening purposes is then the probability of building the project. De facto, pricing in one country is independent of pricing in the other and obeys only to the local redistributive concerns. However, the probability of cancelling the project must be increased since this is now the only way that countries can be screened apart. Inefficiencies in bargaining are then more severe.

Finally, we also analyze the case where the IA has also some redistributive concerns and wants to promote either the welfare of poor countries as a whole, or at a more disaggregated level, the welfare of poor individuals within countries. Those concerns for redistribution reinforce inefficiencies. Far from helping countries in promoting the wellbeing of the poorest agents, those concerns may force to cancel international projects more often and to distort consumption even more.

Our paper lies at the intersection of two different trends of the literature: on the one hand, the traditional analysis of public good mechanisms under informational constraints; on the other hand, the recent and growing game theoretic literature on transnational public goods. Following Groves (1973), the seventies have witnessed important innovations in the design of collective decision mechanisms to mitigate the

\footnotetext{
${ }^{6}$ In a sense to be defined below.
} 
costs of the free-rider problem. Green and Laffont (1977a) characterized mechanisms implementing the first-best production of public goods. Green and Laffont (1977b) and Walker (1980) showed that no such mechanism exists which is budget-balanced. D'Aspremont and Grard-Varet (1979) and Arrow (1979) showed that, by weakening the equilibrium concept from dominant strategy to Bayesian-Nash, budget-balance can be achieved. However, Laffont and Maskin (1979) proved that it was often impossible to satisfy also interim individual rationality constraints. Only more recently, some authors have turned to the second-best problem of designing a collective decision mechanism which maximizes expected social welfare under incentive, participation and budget-balanced constraints. The main contributions along those lines are due to Ledyard and Palfrey (1999), Rob (1989) and Mailath and Postlewaite (1990). These papers are concerned with individual agents and have not developed the hierarchical and nested information structure that is necessary to fully understand the redistributive and allocative consequences of transnational public goods. On the other hand, transnational (or more generally global) public goods have been analyzed in game theoretic environments by Arce and Sandler (2002) and Sandler (1998, 2001). The focus of this literature is on the role of IAs as mediators who provide communication devices, expanding from Nash to correlated set of equilibria of contribution games. We borrow from this literature the idea that IAs play a fundamental role in designing collective mechanisms but we put at the core of the analysis the informational constraints that those IAs face in doing so.

Section 2 describes the model. In Section 3, we derive the important benchmark where the preferences for redistribution in both countries are common knowledge. Asymmetric information on those preferences is introduced in Section 4. Participation constraints of the countries are taken into account in Section 5, which is central to the paper. In Section 6 , we investigate what happens when countries keep control of the pricing schemes. The consequences of having an IA with some redistributive concerns are analyzed in Section 7. Section 8 summarizes our main findings and discusses some extensions of the basic model. Proofs are relegated in the Appendices.

\section{The model}

\subsection{Preferences and technology}

We consider two countries $C_{i}(i=1,2)$ with their respective governments $G_{i}$. Those countries want to build a common infrastructure. Examples of such infrastructures are a common transportation or telecommunication network, a common power grid, a common nuclear or hydroelectricity plant. Each country is unable to finance alone such a large-scale project whose cost is $F$. Both countries have thus to contribute to the financing of a public good which has a $0-1$ nature: building or not the infrastructure. ${ }^{7}$

\footnotetext{
7 Note that there is no alternative to the common infrastructure. The next-best infrastructure gives zero payoff to each agent. This assumption simplifies significantly the analysis.
} 
In each country, there is a continuum of agents with mass one. These agents may have different preferences for the private use of the infrastructure. Their utility function over the quantity $x$ of private consumption ${ }^{8}$ and the corresponding payment made $t$ is:

$$
U=\theta v(x)-t
$$

where $v(\cdot)$ is increasing and concave with the Inada conditions being satisfied $\left(v^{\prime}>0, v^{\prime \prime}<0\right.$, $\left.v^{\prime}(0)=+\infty, v^{\prime}(+\infty)=0\right)$. Introducing the private use of the infrastructure is required to understand how pricing within each country is affected by the external constraints imposed by the collective decision mechanism.

The parameter $\theta$ represents the intensity of an agent's preferences. For instance, $\theta$ may reflect the agent's wealth. With this interpretation, richer people are also the most eager to use the infrastructure. $\theta$ belongs to the set $\Theta=\{\underline{\theta}, \bar{\theta}\}$ (we denote by $\Delta \theta=\bar{\theta}-\underline{\theta}$ the spread of the distribution) with respective probabilities $1-v$ and $v$. We will assume that $\underline{\theta}>(v / 1-v)$ $\Delta \theta$ to always ensure a positive consumption in all the second-best environments described below.

For further references, it is useful to define the first-best surplus of an agent with type $\theta$ consuming $x$ units of services provided by the infrastructure as $S(\theta, x)=\theta v(x)-c x-F / 2$, where $c$ is the constant marginal cost of using the infrastructure and $F / 2$ is the share of the fixed cost that should be paid by this agent. ${ }^{9}$ Of course, efficiency is characterized by the following first-best consumption $x^{*}(\theta)$ for an agent with type $\theta$ :

$$
\theta v^{\prime}\left(x^{*}(\theta)\right)=c .
$$

$G_{i}$ maximizes a weighted sum of $C_{i}$ inhabitants' utilities. Denoting by $U_{i}(\theta)$ the utility of an agent with preferences $\theta, G_{i}$ 's objective function is:

$$
\alpha_{i} v U_{i}(\bar{\theta})+\left(1-\alpha_{i} v\right) U_{i}(\underline{\theta}) .^{10}
$$

where $\alpha_{i}<1$ is a non-negative parameter representing $G_{i}$ 's preferences for redistribution. As $\alpha_{i}$ decreases, the concerns for redistribution towards the poor are more pronounced. In the limiting case where $\alpha_{i}=0$, the government is Rawlsian and cares only about the poor with the smallest utility level. When $\alpha_{i}$ is close to $1, G_{i}$ behaves instead as a pure expected utility maximizer concerned only with efficiency.

To further stress the trade-off between efficiency and redistributive concerns of the government, it is useful to rewrite this objective function as:

$$
v U_{i}(\bar{\theta})+(1-v) U_{i}(\underline{\theta})-v\left(1-\alpha_{i}\right)\left(U_{i}(\bar{\theta})-U_{i}(\underline{\theta})\right) .
$$

\footnotetext{
8 This consumption can be viewed as the amount of electricity consumed if the infrastructure is a power grid or a hydroelectricity plant or the number of phone calls for a telecommunication network.

9 For simplicity, we assume that the two symmetric countries share equally the fixed-cost and finance that amount with lump-sum taxation in a first-best environment.

10 Ledyard and Palfrey (1999) have analyzed such objective functions in the case of a pure public good produced for a single country. Maximization of this objective under incentive constraints yields an interim efficient allocation in the sense of Holmstrm and Myerson (1983).
} 
Countries may differ with respect to the weights $\alpha_{i}$. We assume that the $\alpha_{i}$ s are i.i.d in $\{\underline{\alpha}$, $\bar{\alpha}\}$ (where $0 \leq \underline{\alpha}<\bar{\alpha}<1$ ) with respective probabilities $1-q$ and $q$. We denote by $\Delta \alpha=\bar{\alpha}-\underline{\alpha}$ the spread of this distribution. This can be viewed as a proxy for the maximal degree of polarization between those countries. For technical reasons, we will assume that $\underline{\alpha}>(q / 1-q)$ $\Delta \alpha$ so that the virtual preferences for redistribution defined below remain positive.

\subsection{Asymmetric information}

Asymmetric information plays a role at two levels: within and across countries.

\subsubsection{Within each country}

Agents cannot be discriminated directly on the basis of their tastes which is their private information, but only with respect to their individual consumptions. Pricing has to be incentive compatible and satisfy the self-selection constraints of the different types of consumers. The government's concerns for redistribution lead us to define in a rather standard way ${ }^{11}$ the virtual surplus $\tilde{S}\left(\theta, x, \alpha_{i}\right)$ of an agent with type $\theta$ consuming $x$ units as respectively his true first-best surplus if $\theta=\bar{\theta}$ and a modified surplus

$$
\tilde{S}\left(\underline{\theta}, x, \alpha_{i}\right)=\left(\underline{\theta}-\frac{v}{1-v}\left(1-\alpha_{i}\right) \Delta \theta\right) v(x)-c x-\frac{F}{2}
$$

when $\theta=\underline{\theta}$.

Note that the virtual surplus depends explicitly on $\alpha_{i}$ and that it is lower than the first-best surplus both in absolute terms but also at the margin. If the country with preferences $\alpha_{i}$ were able to self-finance the infrastructure, it is these virtual surpluses which would be maximized for both types of consumers. A poor agent would thus consume a second-best amount:

$$
\left(\underline{\theta}-\frac{v}{1-v}\left(1-\alpha_{i}\right) \Delta \theta\right) v^{\prime}\left(\tilde{x}\left(\underline{\theta}, \alpha_{i}\right)\right)=c,
$$

whereas a rich one would still consume the first-best quantity $\tilde{x}\left(\bar{\theta}, \alpha_{i}\right)=x^{*}(\bar{\theta})$. Note that $\tilde{S}(\theta, \tilde{x}(\theta, \bar{\alpha}), \bar{\alpha})>\tilde{S}(\theta, \tilde{x}(\theta, \underline{\alpha}), \underline{\alpha})$ because $\Delta \alpha>0$.

Within each country, the poor consume less than the first-best. Indeed, ensuring incentive compatibility for the rich requires such a distortion to compensate for the redistribution, which is achieved through pricing. A $\alpha$-government which is the less concerned with redistribution distorts less the consumption of the poor. This also implies that the $\alpha$-country prefers a less egalitarian distribution of utilities than the $\underline{\alpha}$-one.

\subsubsection{Across countries}

The government and the agents within a given country $C_{i}$ have private information on $\alpha_{i}{ }^{12}$ That assumption can be motivated on several grounds. First, it may capture the fact that

\footnotetext{
11 See Ledyard and Palfrey (1999) for instance.

12 Assuming that agents know the redistributive concerns of the government simplifies the presentation and avoids some technicalities associated with solving an informed principal problem which would appear if only $G_{i}$ was privately informed on $\alpha_{i}$. Note nevertheless that this informed principal problem would not be an issue because we are in a context of private values where the principal's type does not enter directly into the preferences of the agent and all utilities are quasi-linear. As in Maskin and Tirole (1990), the same contractual outcome as under complete information on $\alpha_{i}$ within the country would be obtained.
} 
the political process within each country may not be completely transparent for outsiders. For instance, the government of a given country may be more or less biased towards the rich depending on its degree of corruption or the latter group's political influence. Second, the linear specification of the government's preferences can be viewed as a tractable way of introducing a trade-off between efficiency and redistribution in a model with quasi-linear utility functions as argued by Ledyard and Palfrey (1999). ${ }^{13}$ Asymmetric information on the preferences for redistribution can thus be viewed as a proxy for asymmetric information on the budget constraint faced by the country. This seems a highly plausible assumption in the case of LDCs where inefficiencies in the tax systems or in national accounting procedures are likely to throw a veil on the budget constraint. With this interpretation of our model in mind, a government's incentives to lie on its concerns for redistribution can be viewed as coming from its incentives to lie on its true budget constraint and thus on how much it can contribute to the joint project.

For completeness, we provide in Appendix A a simple model which endogenizes simultaneously our modeling choices of objective functions and asymmetric information. In this simple model, we show that a benevolent government putting a priori an equal weight on all different types of agents in its objective function would actually behave as in Eq. (3) because of asymmetric information on the agents' tastes. Then $\alpha_{i}$ reflects the shadow cost of the country's budget constraint. Private information on budget is thus akin to private information on $\alpha_{i}$. In what follows, we will thus assimilate a rich (resp. poor) country as having preferences for redistribution given by $\bar{\alpha}$ (resp. $\underline{\alpha}$ ) but our model allows for alternative interpretations. More generally, a $\alpha$-country is less concerned with redistribution than a $\underline{\alpha}$-one.

\subsection{Timing}

The timing of the game is as follows. First, agents in each country $C_{i}$ learn their individual tastes $\theta$. Government $G_{i}$ and agents in $C_{i}$ learn also the preferences for redistribution $\alpha_{i}$. Second, the IA proposes to both governments a mechanism to finance the common project. Acceptance can be mandatory or not. Third, both governments choose simultaneously how much to contribute to the mechanism and which prices they would like to charge to consumers. Lastly, agents choose their consumption and pay the corresponding price.

\subsection{Mechanisms for collective decision}

An international agency (IA) ${ }^{14}$ acts as a third-party offering to both countries a collective decision mechanism to share the fixed-cost of the infrastructure and

13 This tractability is key to embed the model of intra-country redistribution into a transnational context. It avoids having to handle a true social welfare function defined over the utilities of different types and having to fully endogenize the shadow cost of public funds. In such a more complete and may be more realistic model, the degree of inequality aversion of the government generally depends on the budget constraint it faces (see Martimort, 2001 for a model along these lines). When inequality aversion decreases with wealth, a poorer country will have more incentives to redistribute. A poor (resp. rich) country can thus be viewed as one having a parameter $\underline{\alpha}$ (resp. $\alpha$ ).

14 Like the World Bank and its regional counterparts, the Asian Development Bank, the Inter-American Development Bank, and the African Development Bank. 
determine the prices charged to the different types of consumers in each country. The IA provides thus an institutional solution to the bargaining problem between the countries. The IA acts indeed as a "mediator" in the sense of Myerson and Sattherwaite (1983). It collects contributions, commits to build the infrastructure with some probability and recommends prices. It does not bring any external funds. ${ }^{15,16}$ Accordingly, this third-party is a benevolent welfare maximizer putting an equal weight on each country in its objective function. Had both governments being efficiency maximizers $\left(\alpha_{i}=1\right)$ the IA would be concerned with ex ante efficiency. Instead, the existence of redistributive concerns at the local level means that the IA is concerned with ex ante constrained efficiency where the term constrained should be understood as capturing those local redistributive concerns only.

Given the two-tier asymmetric information postulated (both on tastes but also on the countries' preferences for redistribution), a mechanism stipulates first the probability $p(\cdot)$ of building the infrastructure and the overall contribution of each country $T_{i}(\cdot)$ as a function of the reports $\hat{\alpha}=\left(\hat{\alpha}_{1}, \hat{\alpha}_{2}\right)$ made by both countries on their preferences for redistribution. Second, given those reports, the mechanism stipulates also what should be the price paid $t_{i}(\cdot, \hat{\alpha})$ and the consumptions $x_{i}(\cdot, \hat{\alpha})$ of each agent in $C_{i}$ as a function of his own report $\hat{\theta}$ on his taste parameter. A mechanism is thus a vector of functions $\left\{p(\hat{\alpha}) ; T_{i}(\hat{\alpha}) ; t_{i}(\hat{\theta}, \hat{\alpha}) ; x_{i}(\hat{\theta}, \hat{\alpha})\right\}$. Using the Revelation Principle, there is no loss of generality in looking for direct mechanisms where truthful reports are optimal strategies. $^{17}$

To cover the overall contribution of a given country plus the cost of producing private services within that country, the prices charged to both types of consumers must satisfy for any profile $\alpha$ of preferences:

$$
T_{i}(\alpha)=p(\alpha) \underset{\theta}{E}\left(t_{i}(\theta, \alpha)-c x_{i}(\theta, \alpha)\right) .
$$

This expression shows that the contribution of a country to the project is covered by the prices paid by local consumers. Of course, these prices are only charged conditionally on the fact that the project is realized. ${ }^{18}$

15 It is easy to introduce some amount of external financing in our framework. If we denote by $F^{\prime}$ the fixedcost of the project, and by $K$ the amount of external funds provided, our model could account for this extension provided that $F^{\prime}-K$ is now viewed as the fixed-cost of the project.

16 Other roles of the IA could be introduced. Schiff and Winters (2002) stress the role of international organizations in inducing more cooperative outcomes by fostering trust and providing expertise on state-of-the-art technology, engineering and financing. Section 7 analyzes the case of an IA with redistributive concerns.

17 Because $\alpha_{i}$ is known by both the government $G_{i}$ and the residents of $C_{i}$, the IA could use a more complex revelation mechanism eliciting costlessly this commonly known but non-verifiable information (see Maskin, 1999). To rule out those mechanisms, we assume that the IA is unable to communicate directly with individuals.

18 Note that the contribution defined by Eq. (5) is paid upfront, i.e., before the realization of the project or not. To balance the budget of each government, we must ensure that this contribution is covered by what can be raised from consumers net of the cost of using the infrastructure. Note also that there is no need to have countries pay different amounts depending on whether the project is built or not in this context with quasi-linear utility functions. Only the upfront payment matters for providing incentives to the countries for revealing their information. 


\subsubsection{Notations}

Given the symmetry of the model, we focus on symmetric mechanisms, omit indices and sometimes denote overall contributions as $\bar{T}=T_{i}(\bar{\alpha}, \bar{\alpha}), \hat{T}_{1}=T_{1}(\bar{\alpha}, \underline{\alpha})=T_{2}(\underline{\alpha}$, $\bar{\alpha}), \hat{T}_{2}=T_{1}(\underline{\alpha}, \bar{\alpha})=T_{2}(\bar{\alpha}, \underline{\alpha})$ and $\underline{T}=T_{i}(\underline{\alpha}, \underline{\alpha})$. The probabilities of building the infrastructure are also written as $\bar{p}=p(\bar{\alpha}, \bar{\alpha}), \hat{p}=p(\bar{\alpha}, \underline{\alpha})=p(\underline{\alpha}, \bar{\alpha})$ and $\underline{p}=p(\underline{\alpha}, \underline{\alpha})$. Finally, still for a symmetric mechanism, we denote consumptions by $\bar{x}_{i}(\theta, \alpha)=x(\theta, \alpha)$. Similar conventions hold for the prices $t_{i}(\cdot)$ and the utilities $U_{i}(\cdot)$.

The mechanism works as follows. First, countries report their preferences for redistribution, pay a contribution and are offered a menu of possible prices and consumptions for agents within the country. Second, agents choose within the proposed menus how much they want to consume and pay the corresponding prices. This implies that their incentive compatibility constraints are written for a given pair of truthful reports $\alpha=\left(\alpha_{1}, \alpha_{2}\right) .{ }^{19}$ Although, there is a single grand-contract offered by the IA, that mechanism can easily be interpreted as a nexus of bilateral contracts, one between countries and other ones within each country defining the pricing scheme. The key thing to notice is that those contracts are cooperatively designed by the IA, except in Section 6.

\section{Common knowledge on countries' preferences}

Let us first suppose that the IA has complete information on the countries' profile of preferences for redistribution $\alpha=\left(\alpha_{1}, \alpha_{2}\right) .{ }^{20}$

Within each country, the agents' incentive constraints can thus be expressed in terms of the utility $U_{i}(\theta, \alpha)$ they get and their consumptions $x_{i}(\theta, \alpha)$. These constraints are respectively

$$
\begin{aligned}
U_{i}(\bar{\theta}, \alpha) & =p(\alpha)\left(\bar{\theta} v\left(x_{i}(\bar{\theta}, \alpha)\right)-t_{i}(\bar{\theta}, \alpha)\right) \geq p(\alpha)\left(\bar{\theta} v\left(x_{i}(\underline{\theta}, \alpha)\right)-t_{i}(\underline{\theta}, \alpha)\right) \\
& =U_{i}(\underline{\theta}, \alpha)+p(\alpha) \Delta \theta v\left(x_{i}(\underline{\theta}, \alpha)\right) .
\end{aligned}
$$

and

$$
\begin{aligned}
U_{i}(\underline{\theta}, \alpha) & =p(\alpha)\left(\underline{\theta} v\left(x_{i}(\underline{\theta}, \alpha)\right)-t_{i}(\underline{\theta}, \alpha)\right) \geq p(\alpha)\left(\underline{\theta} v\left(x_{i}(\bar{\theta}, \alpha)\right)-t_{i}(\bar{\theta}, \alpha)\right) \\
& =U_{i}(\bar{\theta}, \alpha)-p(\alpha) \Delta \theta v\left(x_{i}(\bar{\theta}, \alpha)\right) .
\end{aligned}
$$

As it is standard in two-type adverse selection models, ${ }^{21}$ the relevant (binding) constraint is that of the rich agent $\bar{\theta}$, namely Eq. (6), whereas Eq. (7) will be slack at the optimum as it can be checked ex post on the solution.

19 This is akin to a dominant strategy requirement when writing the agents' incentive constraints. Alternatively, we could assume that the agents and the countries report simultaneously. Given that an agent in $C_{i}$ does not know $\alpha_{-i}$, we would have to focus on Bayesian incentive compatibility constraints. As shown in Mookherjee and Reichelstein (1992), there is nevertheless no loss of generality in focusing on dominant strategy implementation as long as the decision rule is monotonic, a property which holds in the sequel.

20 Alternatively, this can be viewed as a setting where the IA uses revelation schemes a la Maskin making both each government $G_{i}$ and the inhabitants of $C_{i}$ report the commonly known piece of information $\alpha_{i}$.

21 See Laffont and Martimort (2002, Chap. 2). 
Contributions must cover the (expected) cost of building the project under any profile of preferences:

$$
\sum_{i=1}^{2} T_{i}(\alpha) \geq p(\alpha) F
$$

We rewrite this constraint using Eq. (5) and the definitions of $U_{i}(\theta, \alpha)$ given above as:

$$
\sum_{i=1}^{2}\left(p(\alpha) \underset{\theta}{E}\left(S\left(\theta, x_{i}(\theta, \alpha)\right)-U_{i}(\theta, \alpha)\right) \geq 0 .\right.
$$

Intuitively, the sum of expected surpluses in both countries computed for the consumption profile $x_{i}(\theta, \alpha)$ must equal the sum of utilities redistributed within each country.

Being given the preferences profile $\alpha$, the IA solves the following problem:

$$
(\mathrm{TP})^{\mathrm{FB}}: \max _{\left\{p(\cdot) ; U_{i}(\cdot), x_{i}(\cdot), \tilde{V}_{i}(\cdot)\right\}} \sum_{i=1}^{2} \tilde{V}_{i}(\alpha),
$$

subject to Eqs. (6), (7) and (9) and

$$
\tilde{V}_{i}(\alpha)=\alpha_{i} v U_{i}(\bar{\theta}, \alpha)+\left(1-\alpha_{i} v\right) U_{i}(\underline{\theta}, \alpha) .
$$

In Appendix A, we show that solving (TP) ${ }^{\mathrm{FB}}$ amounts in fact to solving the following simpler problem:

$$
(\mathrm{TP})^{\mathrm{FB} *}: \max _{\left\{p(\cdot), x_{i}(\cdot)\right\}} p(\alpha)\left\{\sum_{i=1}^{2} \underset{\theta}{E}\left(\tilde{S}\left(\theta, x_{i}(\theta, \alpha), \alpha_{i}\right)\right)\right\} .
$$

This simple objective aggregates the rich agents' incentive constraint (6) and the budget-balanced constraint (9) to get a more compact expression which depends only on the probability of building the project and the consumption profiles conditionally on its realization. A first trivial observation is that the IA uses together these two tools to maximize the sum of expected welfares in both countries.

Because governments in both countries have some redistributive concerns, the IA considers in fact the sum of expected virtual surpluses in both countries and not the sum of their true surpluses as an objective. To focus on the most interesting case from an economic point of view, we assume that the following conditions hold:

$$
\begin{aligned}
& \underset{\theta}{E}(\tilde{S}(\theta, \tilde{x}(\theta, \bar{\alpha}), \bar{\alpha}))+\underset{\theta}{E}(\tilde{S}(\theta, \tilde{x}(\theta, \underline{\alpha}), \underline{\alpha}))>0, \\
& \underset{\theta}{E}(\tilde{S}(\theta, \tilde{x}(\theta, \underline{\alpha}), \underline{\alpha}))<0 .
\end{aligned}
$$

These conditions establish that the infrastructure must be built as soon as at least one of the countries is not too much concerned by poverty. They define in fact the constrained efficient probabilities of realizing the project, i.e., the optimal probabilities 
when a utilitarian welfare maximizer aggregates the countries' welfare which take into account the consumers' incentive constraints. (H1) and (H2) also imply that the decision of building or not the infrastructure is conditional on the exact profile of preferences for redistribution. A precise knowledge of those preferences is needed to fine-tune the policy of the IA. This fine-tuning will be the source of some problems under asymmetric information on the $\alpha_{i}$ s. Clearly, if it was optimal to do the project whatever the countries' preferences, asymmetric information on those preferences would not be an issue. It would be enough to have both countries paying the same amount (something less or equal to the expected virtual surplus of the $\underline{\alpha}$-one) to get enough cash to finance the project.

Proposition 1 summarizes the optimal mechanism offered by the benevolent IA.

Proposition 1. Assume that the preferences profile $\alpha$ is common knowledge and that conditions (H1) and (H2) both hold. Then, the optimal mechanism for collective decision is characterized as follows.

- The decision to build or not the infrastructure is constrained efficient. Probabilities of building the infrastructure are given by $\bar{p}^{*}=\hat{p}^{*}=1$ and $p^{*=0}$.

- The rich agents' incentive constraint (6) is binding as soon as the project is done.

- The rich agents consume always the first-best amount $x^{*}(\bar{\theta})$, whereas the poor agents in $C_{i}$ consume the second-best quantity $\tilde{x}\left(\underline{\theta}, \alpha_{i}\right)$ as soon as the project is built.

- Pricing in any country depends only on the local redistributive concerns.

Of course, the redistributive concerns of each government affect the pricing schemes suggested by the IA. Even though the IA puts an equal weight on both countries' welfare, governments are not efficiency maximizers, and there will remain some distortions in consumption and pricing away from the first-best. However, there are no more distortions than those implied by the local preferences for redistribution.

In each country, the rich consume at the first-best level. Instead, consumption is still downward distorted for the poor and takes the same second-best value as if each country was able to self-finance the project. Indeed, satisfying the incentive compatibility constraint (6) requires to give more utility to a $\bar{\theta}$-agent than to a $\underline{\theta}$-one. Since governments are averse to inequality between consumers, doing so is costly. This cost is reduced by decreasing the consumption of the poor as it can be seen from Eq. (6). ${ }^{22}$

Since pricing within each country only depends on the local preferences for redistribution, there is a complete dichotomy between pricing and the decision to build or not the project which, instead, depends on the preferences for redistribution in both countries. This dichotomy may be lost under asymmetric information as we will see below. In that case, there might appear an endogenous link between prices within both countries. Any departure from the dichotomy result can thus be best understood as coming from the impossibility for a given country to charge prices in the same way as if

\footnotetext{
22 Of course, a utilitarian government (corresponding to the limiting case $\bar{\alpha}=1$ ) does not care about the distribution of utilities within the country. Then, the rich agents' incentive constraint becomes costless and the poor consume also the first-best consumption $x^{*}(\underline{\theta})$.
} 
self-financing was feasible. What Proposition 1 shows then is that the external constraints that a collective agreement imposes on a country do not modify the redistributive concerns of each country as long as the preferences profile for redistribution is common knowledge.

\section{Asymmetric information on countries' preferences: constrained efficiency}

We now consider the case where the IA is uninformed on the $\alpha_{i}$ s. To understand precisely the distortions involved by this added tier of asymmetric information, it is useful to see the mechanism offered by the IA as specifying the contribution and the distribution of utility for each country. Letting the governments report their preferences amounts to having them choose within a menu of possible distributions of utility. This new formulation is more tractable to express the various constraints of our problem and illuminates the true nature of the decisions of those governments. In fine, governments are not really interested in the prices charged to consumers per se but care about the distribution of utility that those prices induce.

Let us turn to a description of the governments' Bayesian incentive constraints:

$$
\begin{aligned}
V_{i}(\bar{\alpha}) & =\underset{\alpha_{-i}}{E}\left(\underset{\theta}{E}\left(U_{i}\left(\theta, \bar{\alpha}, \alpha_{-i}\right)\right)-v(1-\bar{\alpha})\left(U_{i}\left(\bar{\theta}, \bar{\alpha}, \alpha_{-i}\right)-U_{i}\left(\underline{\theta}, \bar{\alpha}, \alpha_{-i}\right)\right)\right) \\
& \geq \underset{\alpha_{-i}}{E}\left(\underset{\theta}{E}\left(U_{i}\left(\theta, \underline{\alpha}, \alpha_{-1}\right)\right)-v(1-\bar{\alpha})\left(U_{i}\left(\bar{\theta}, \underline{\alpha}, \alpha_{-i}\right)-U_{i}\left(\underline{\theta}, \underline{\alpha}, \alpha_{-i}\right)\right)\right) \\
& =V_{i}(\underline{\alpha})+v \Delta \alpha \underset{\alpha_{-i}}{E}\left(U_{i}\left(\bar{\theta}, \underline{\alpha}, \alpha_{-i}\right)-U_{i}\left(\underline{\theta}, \underline{\alpha}, \alpha_{-i}\right)\right) .
\end{aligned}
$$

and

$$
\begin{aligned}
V_{i}(\underline{\alpha}) & =\underset{\alpha_{-i}}{E}\left(\underset{\theta}{E}\left(U_{i}\left(\theta, \underline{\alpha}, \alpha_{-i}\right)\right)-v(1-\underline{\alpha})\left(U_{i}\left(\bar{\theta}, \underline{\alpha}, \alpha_{-i}\right)-U_{i}\left(\underline{\theta}, \underline{\alpha}, \alpha_{-i}\right)\right)\right) \\
& \geq \underset{\alpha_{-i}}{E}\left(\underset{\theta}{E}\left(U_{i}\left(\theta, \bar{\alpha}, \alpha_{-1}\right)\right)-v(1-\underline{\alpha})\left(U_{i}\left(\bar{\theta}, \bar{\alpha}, \alpha_{-i}\right)-U_{i}\left(\underline{\theta}, \bar{\alpha}, \alpha_{-i}\right)\right)\right) \\
& =V_{i}(\bar{\alpha})-v \Delta \alpha \underset{\alpha_{-i}}{E}\left(U_{i}\left(\bar{\theta}, \bar{\alpha}, \alpha_{-i}\right)-U_{i}\left(\underline{\theta}, \bar{\alpha}, \alpha_{-i}\right)\right) .
\end{aligned}
$$

In what follows, the only relevant (binding) incentive constraint is Eq. (11). It says that a rich country must be prevented from reporting being poor. By doing so, it indeed pays a smaller contribution. Furthermore, because of asymmetric information on tastes, a given level of aggregated welfare can only be implemented by imposing some costly inequality within the country. A rich country finds it easier to bear such inequality. By mimicking a poor country, a rich one can thus save on the redistribution costs. The less egalitarian the distribution of utility in the poor country, the harder it is to satisfy the incentive constraint (11).

Note also that, when the IA has the strongest ability to enforce the mechanism, the countries' participation constraints do not matter. Acceptance of the mechanism is mandatory. 
The IA proposes an incentive-compatible mechanism before knowing the realizations of the $\alpha_{i}$ s. Using the definition of the $V_{i}(\cdot)$ and the fact that the IA maximizes now the sum of expected welfares in both countries, IA's problem becomes:

$$
(\mathrm{TP})_{0}: \max _{\left\{p(\cdot) ; x_{i}(\cdot) ; U_{i}(\cdot) ; V_{i}(\cdot)\right\}} \sum_{i=1}^{2} E\left(V_{\alpha_{i}}\left(V_{i}\right)\right),
$$

subject to (Eqs. (6), (7), (8), (11) and (12)).

We also show in Appendix A that (TP $)_{0}$ can be expressed in a more compact way as: ${ }^{23}$

$$
(\mathrm{TP})_{0} *: \max _{\left\{p(\cdot), x_{i}(\cdot), V_{i}(\cdot)\right\}} E\left\{p(\alpha)\left(\sum_{i=1}^{2} E\left(\tilde{\theta}\left(\theta, x_{i}(\theta, \alpha), \alpha_{i}\right)\right)\right)\right\}
$$

subject to Eqs. (11) and (12), and

$$
\sum_{i=1}^{2} \underset{\alpha_{i}}{E}\left(V_{i}\left(\alpha_{i}\right)\right)=\underset{\alpha}{E}\left\{p(\alpha)\left(\sum_{i=1}^{2} \underset{\theta}{E}\left(\tilde{S}\left(\theta, x_{i}(\theta, \alpha), \alpha_{i}\right)\right)\right)\right\}
$$

Proposition 2. Assume that the preferences profile $\alpha$ is private information and that conditions (H1) and (H2) both hold. Then, the optimal mechanism is constrained efficient. It entails the same probabilities of building the infrastructure, namely $\bar{p}^{*}=\hat{p}^{*}=1$ and $p^{*}=0$ and the same second-best consumption levels as when preferences are common knowledge.

The problem of building the infrastructure can be viewed as a public good problem along the lines of D'Aspremont and Grard-Varet (1979) and Arrow (1979). The work of these authors shows that one can design a Bayesian incentive compatible and ex post budget-balanced mechanism which implements the ex ante efficient outcome. The same logic applies here. However, there is an added complexity. The mechanism not only stipulates whether the common infrastructure is built or not but it also defines prices in both countries. On top of that, the outcome which maximizes the sum of expected welfare in both countries is not first-best but constrained efficient because of the existing redistributive concerns of both governments. In an Appendix, we show that there exists a whole continuum of mechanisms and contributions (which can be indexed by the level of aggregate welfare in a poor country) which achieve this outcome. ${ }^{24}$ Given these contributions, the pricing schemes within each country still give to the agents incentives to truthfully report their tastes. Again, the distortions for consumption within each country are totally disentangled from the decision rule on whether to build or not the infrastructure. The dichotomy between pricing at the local level and the international collective decision is maintained.

23 The expression below no longer contains the contributions made by countries as variables. We show in Appendix A that, reciprocally, one can find transfers that implement a corresponding allocation of consumptions and aggregate welfare for each type of country.

24 Among these mechanisms, a focal one might be the so-called pay-the-externality mechanism stressed by D'Aspremont and Grard-Varet (1979) and Arrow (1979). This mechanism is such that each country pays a contribution equal to the expected shift in welfare that the other incurs following a change in its own report. 


\section{Asymmetric information on countries' preferences and countries' participation constraints}

Even though the results in proposition seem attractive on normative grounds and suggest that asymmetric information may not be such an obstacle to investment, the previous mechanisms suffer from a serious flaw since the countries' participation constraints may not be satisfied. In fact, accepting the international agreement is a sovereign act. To do so, each country must get more than its payoff (that we have exogenously normalized at zero) without any infrastructure.

Note that the symmetric mechanism which is the solution to (TP)* defines only the expected welfare $q V(\bar{\alpha})+(1-q) V(\underline{\alpha})$ of a given country. ${ }^{25}$ As already stressed, there exists a whole range of possible values of $(V(\underline{\alpha}), V(\bar{\alpha}))$ which satisfy the incentive constraints $(11)$ and (12) and correspond to the same expected welfare. One may wonder whether, within this range, there exist some pairs $\left(V_{i}(\underline{\alpha}), V_{i}(\bar{\alpha})\right)$ satisfying also the following interim participation constraints of countries:

$$
\begin{aligned}
& V_{i}(\bar{\alpha}) \geq 0, \\
& V_{i}(\underline{\alpha}) \geq 0 .
\end{aligned}
$$

In fact, with those participation constraints, constrained efficiency cannot always be achieved.

Proposition 3. The constrained efficient decision-rule $\left(\bar{p}^{*}, \hat{p}^{*}, p^{*}\right)$ and the second-best levels of consumption $\tilde{x}\left(\theta, \alpha_{i}\right)$ can no longer be implemented when the countries' interim participation constraints (14) and (15) must be satisfied if the following condition holds:

$$
\begin{aligned}
& 2 q^{2} \underset{\theta}{E}(\tilde{S}(\theta, \tilde{x}(\theta, \bar{\alpha}), \bar{\alpha}))+2 q(1-q)\left(E_{\theta}(\tilde{S}(\theta, \tilde{x}(\theta, \bar{\alpha}), \bar{\alpha}))+\underset{\theta}{E}(\tilde{S}(\theta, \tilde{x}(\theta, \underline{\alpha}), \underline{\alpha}))\right) \\
& \quad<2 q^{2} v \Delta \alpha \Delta \theta v(\tilde{x}(\underline{\theta}, \underline{\alpha})) .
\end{aligned}
$$

This condition says that the sovereignty of countries is a source of inefficiency whenever the aggregated welfare computed for the constrained efficient outcome is smaller than the cost of inducing information revelation from the $\bar{\alpha}$-countries. Condition (16) is related to an earlier result due to Laffont and Maskin (1979) who proved that Bayesian incentive compatibility, efficiency, budget balance and individual rationality may be incompatible, and to Makowski and Mezzetti (1994) and Williams (1999) characterizations of the individual rationality payoffs which reconcile all those requirements.

To induce revelation of the country's preferences, the IA must propose an unequal distribution of expected welfares across different types of countries. The $\bar{\alpha}$-country

\footnotetext{
25 We omit indices because of symmetry.
} 
receives more than the $\underline{\alpha}$-one. From Eq. (11), the induced inequality is greater when the IA maintains an efficient probability of building the infrastructure and sets $\hat{p}^{*}=1$. Indeed, if the constrained efficient outcome was implemented, Eq. (11) could be written as:

$$
V_{i}(\bar{\alpha}) \geq V_{i}(\underline{\alpha})+v q \Delta \alpha \Delta \theta v(\tilde{x}(\underline{\theta}, \underline{\alpha})) \quad \text { for } i \in\{1,2\} .
$$

When $\hat{p}^{*}=1$ and the second-best consumption $\tilde{x}(\underline{\theta}, \underline{\alpha})$ is maintained, the right-hand side of Eq. (17) is rather large meaning that $V_{i}(\underline{\alpha})$ must be significantly lower than $V_{i}(\bar{\alpha})$ to ensure incentive compatibility. The $\underline{\alpha}$-country may thus end up with a negative expected welfare. Satisfying the participation constraint of the $\underline{\alpha}$-country calls for reducing the contribution of this country. This makes the $\bar{\alpha}$-country more eager to mimic the $\underline{\alpha}$-one, hardening thereby its incentive constraint (11). Incentive compatibility at the country level is thus easier to achieve if $\hat{p}$ is distorted downwards and if, when countries have different preferences for redistribution, the consumption of the poor agents within a $\underline{\alpha}$-country is downward distorted below the second-best.

Let us characterize the optimal mechanism. Note that the IA's problem becomes now:

$$
(\mathrm{TP})^{\mathrm{SB}}: \max _{\left\{p(\cdot) ; x_{i}(\cdot) ; U_{i}(\cdot) ; V_{i}(\cdot)\right\}} \sum_{i=1}^{2} \underset{\alpha_{i}}{E}\left(V_{i}\left(\alpha_{i}\right)\right)
$$

subject to Eqs. (6), (7), (9), (11), (12), (14) and (15).

We show in an Appendix that this problem can be simplified to:

$$
(\mathrm{TP})^{\mathrm{SB} *}: \max _{\left\{p(\cdot), x_{i}(\cdot)\right\}} E\left\{p(\alpha)\left(\sum_{i=1}^{2} \underset{\theta}{E}\left(\tilde{S}\left(\theta, x_{i}(\theta, \alpha), \alpha_{i}\right)\right)\right)\right\}
$$

subject to

$$
\begin{aligned}
& E_{\alpha}\left\{p(\alpha)\left(\sum_{i=1}^{2} \underset{\theta}{E}\left(\tilde{S}\left(\theta, x_{i}(\theta, \alpha), \alpha_{i}\right)\right)\right)\right\} \\
& \quad-\sum_{i=1}^{2}\left\{v q \Delta \alpha \Delta \theta \underset{\alpha_{-i}}{E}\left(p\left(\underline{\alpha}, \alpha_{-i}\right) v\left(x_{i}\left(\underline{\theta}, \underline{\alpha}, \alpha_{-i}\right)\right)\right)\right\} \geq 0 .
\end{aligned}
$$

This constraint aggregates the ex post budget-balanced constraint (9), the relevant (binding) incentive constraint (11) of the $\bar{\alpha}$-countries and the participation constraint (15) of a $\underline{\alpha}$-country. Condition (18) simply means that the aggregate welfare over both countries should cover the informational cost of inducing information revelation by the $\bar{\alpha}$-countries. The impact of this constraint is akin to that of a budget-breaking constraint in RamseyBoiteux models. As in those models, allocative distortions are needed to satisfy Eq. (18) and the shadow cost of this constraint plays an important role in the characterization of those distortions.

The optimal mechanism might be quite complex and involve distortions of both the pricing rule and/or the decision to build or not the infrastructure. To obtain clear results 
and highlight conditions such that both kinds of distortions are in fact needed, we will assume that the fixed-cost $F$ is large enough so that the following condition holds:

$$
\begin{aligned}
& 2 q^{2} \underset{\theta}{E}(\tilde{S}(\theta, \tilde{x}(\theta, \bar{\alpha}), \bar{\alpha}))+2 q(1-q)\left(E_{\theta}(\tilde{S}(\theta, \tilde{x}(\theta, \bar{\alpha}), \bar{\alpha}))+\underset{\theta}{E}\left(\tilde{S}\left(\theta, x_{\infty}(\theta, \underline{\alpha}), \underline{\alpha}\right)\right)\right) \\
& \quad<2 v q^{2} \Delta \alpha \Delta \theta v\left(x_{\infty}(\underline{\theta}, \underline{\alpha})\right),
\end{aligned}
$$

where $x_{\infty}(\bar{\theta}, \underline{\alpha})=x^{*}(\bar{\theta})$ and $x_{\infty}(\underline{\theta}, \underline{\alpha})$ is defined by:

$$
\left(\underline{\theta}-\frac{v(1-\underline{\alpha})}{1-v} \Delta \theta-\frac{v q \Delta \alpha}{(1-v)(1-q)} \Delta \theta\right) v^{\prime}\left(x_{\infty}(\underline{\theta}, \underline{\alpha})\right)=c .
$$

Eq. (19) strengthens condition (16) and describes cases where inefficiencies are large. Indeed, there does not exist any modification of the second-best consumption $\tilde{x}(\underline{\theta}, \underline{\alpha})$ which, alone, could ensure that the feasibility condition (18) is satisfied. Playing on the probability of building or not the project is absolutely needed. ${ }^{26}$

Proposition 4. Assume that countries can opt out of the mechanism if they do not get a non-negative expected welfare and that condition (19) holds. The optimal mechanism is characterized as follows.

- The $\underline{\alpha}$-country gets zero expected welfare (its participation constraint (15) is binding).

- The decision to build the infrastructure is distorted with the project being realized less often than when countries' preferences are common knowledge. In particular, when countries are asymmetric, the probability of building the project is positive but always less than one: $\bar{p}^{S B}=\bar{p}^{*}=1, \hat{p}^{S B} \in[0,1], p^{S B}=p^{*}=0$.

- Consumptions in the $\bar{\alpha}=$ countries are still constrained efficient; $x^{S B}\left(\bar{\theta}, \bar{\alpha}, \alpha_{-i}\right)=x^{*}(\bar{\theta})$ and $x^{S B}\left(\underline{\theta}, \bar{\alpha}, \alpha_{-i}\right)=\tilde{x}(\underline{\theta}, \alpha)$ for all $\alpha_{-i}$.

- There is an extra downward distortion of the consumption of the poor in the $\underline{\alpha}-$ countries: $x^{S B}(\bar{\theta}, \underline{\alpha}, \bar{\alpha})=x^{*}(\bar{\theta})$ and $x^{S B}(\underline{\theta}, \underline{\alpha}, \alpha)<\tilde{x}(\underline{\theta}, \underline{\alpha})$. Denoting by $\lambda>0$, the shadow cost of the feasibility constraint (18), we have:

$$
\left(\underline{\theta}-\frac{v(1-\underline{\alpha})}{1-v} \Delta \theta-\frac{\lambda v q \Delta \alpha}{(1+\lambda)(1-v)(1-q)} \Delta \theta\right) v^{\prime}\left(x^{\mathrm{SB}}(\underline{\theta}, \underline{\alpha}, \bar{\alpha})\right)=c .
$$

- Pricing in a poor country depends on the shadow cost $\lambda$ and thus on the redistributive concerns within the rich country which is the only partner with which the project is realized.

We already noticed that the conflict between the incentive constraint of a $\bar{\alpha}$-country and the participation constraint of a $\underline{\alpha}$-one is solved by moving to a policy which is no longer constrained efficient. When countries have asymmetric preferences, the project is no

\footnotetext{
26 When Eq. (19) does not hold but still Eq. (16) holds, we are in cases of intermediate inefficiencies where, depending on the functional forms, a distortion on the decision to build or not the infrastructure may be needed. To get sharper results, we omit these less interesting cases.
} 
longer realized with probability one at the optimum. The optimal mechanism is necessarily random and requires to cancel the project with some positive probability. ${ }^{27,28}$

To solve this conflict, the IA must make the distribution of utilities within the $\underline{\alpha}$-country less attractive to a $\bar{\alpha}$-country. In a $\bar{\alpha}$-country, inequality is less costly than in a $\underline{\alpha}$-one. By mimicking a $\underline{\alpha}$-country, a $\bar{\alpha}$-one reduces its overall contribution but it also chooses a more egalitarian distribution of utilities. When the IA offers to a $\underline{\alpha}$-country a pricing scheme inducing a very egalitarian distribution of utilities, it reduces also the incentives of a $\bar{\alpha}$ country to mimic a $\underline{\alpha}$-one. A more egalitarian distribution of utilities in the $\underline{\alpha}$-country is an optimal response to the informational problem that the IA faces.

From Eq. (21), everything happens thus as if the $\underline{\alpha}$-country had now a positive stronger virtual aversion to inequality $\underline{\tilde{\alpha}}$ defined as:

$$
\underline{\tilde{\alpha}}=\underline{\alpha}-\frac{\lambda q}{(1+\lambda)(1-q)} \Delta \alpha<\underline{\alpha} .
$$

This modification of the redistributive concerns within the $\underline{\alpha}$-country captures how the incentive problem between countries trickles down within the countries themselves. Pricing within a $\underline{\alpha}$-country is distorted to limit consumption by the poor and make them pay less for the infrastructure. In fact, by introducing a participation constraint at the country level, one implicitly gives to the IA a redistributive concern and makes it averse to inequality across countries. These concerns add up to the aversion to inequality within countries themselves to justify more redistributive policies.

At a broader level, the fact that the nested information structure of our model leads to extra distortions away from (constrained) efficiency bears some resemblance with some of the results of the literature on hierarchical contracting. ${ }^{29}$ There, it is shown that taking seriously into account the participation constraints of intermediate layers may increase inefficiency when contracts at different tiers are chosen non-cooperatively. Nevertheless, there remain several important differences with our model. Contrary to this literature, we are concerned with the trade-off between efficiency and redistribution instead of that between efficiency and rent extraction. Also, even though our information structure is nested, the whole economy is ruled through a single mechanism which helps countries to coordinate prices and not as a sequence of bilateral contracts chosen non-cooperatively by the different tiers of the hierarchy. ${ }^{30}$

Interestingly, the consumption $x^{\mathrm{SB}}(\underline{\theta}, \underline{\alpha} \bar{\alpha})$ is always strictly above $x_{\infty}(\underline{\theta}, \underline{\alpha})$ which is obtained by setting $\lambda=+\infty$ into Eq. (21). This ranking captures again the fact that distorting pricing is a useful tool to induce information revelation but it is not sufficient

\footnotetext{
27 In the game theoretic model of Arce and Sandler (2002), the IA acts also as a mediator who enforces a correlated equilibrium where randomness in the outcome improves cooperation. In our framework, that randomness relaxes incentive constraints.

28 Of course, enforcing a random mechanism is a more difficult task than enforcing a deterministic contract but the IA's reputation may play a role in making credible the commitment to such a random outcome.

29 See Melumad et al. (1995), McAfee and McMillan (1995) and Faure-Grimaud et al. (2003) among others.

30 See nevertheless Section 6 for an extension where pricing is decided at the local level without coordination between countries.
} 
alone. One cannot avoid cancelling the project with some positive probability. Moreover, if the project is sometimes canceled, distortions in consumption are not maximal.

Importantly, the dichotomy between the decision to build or not the infrastructure and the pricing rules no longer holds when countries must voluntarily participate to the mechanism. Both pricing and the decision to build or not the project are used altogether to solve the incentive problem at the country level. A lower probability of building the infrastructure when countries are asymmetric relaxes Eq. (18) and makes it less necessary to distort consumption in a poor country. If the project is realized less often, the marginal price paid by the poor for an extra unit of consumption can be reduced with respect to the price that would be charged had the IA committed to always realize the project when the countries have asymmetric preferences.

The shadow cost $\lambda$ of the feasibility constraint (18) plays a crucial role in linking distortions on pricing and consumption and the distortion on the probability of realizing the project. For instance, when condition (16) is almost an equality, i.e., for a fixed-cost which is not too large. ${ }^{31} \lambda$ is small, the probability $\hat{p}$ of building the project in the case of asymmetric preferences is close to one and the distortions in the distribution of utilities within the country are weakened. Pricing in a poor country is almost kept unchanged.

\section{Loss of control on pricing}

We now investigate a less comprehensive contracting environment where the IA can no longer control pricing and let governments choose freely the prices charged to consumers. This choice can thus be viewed as a moral hazard variable not observable by the IA. The economy is no longer ruled through a set of bilateral contracts which are cooperatively set by the IA but by bilateral contractual relationships between and within countries which are no longer as coordinated. There is clearly a loss of control associated to relinquishing control rights on pricing to the national level. This section analyzes the consequences of this loss of control.

First, note that, for any probability of making the project and the overall contribution made by a country, consumptions reflect now only the preferences in this country, and the optimal second-best profile of consumptions specific to each country $\tilde{x}\left(\theta, \alpha_{i}\right)$ is always implemented. Second, the only screening instruments available to the IA are now the contributions and the probability of building the infrastructure. A mechanism in this environment is of the form $\left\{p(\hat{\alpha}) ; T_{i}(\hat{\alpha})\right\}$.

Let us first redefine the expected welfare in country $C_{i}$ as:

$$
V_{i}\left(\alpha_{i}\right) \underset{\alpha_{-i}}{E}\left(-T_{i}(\alpha)+p(\alpha) \underset{\theta}{E}\left(\tilde{S}\left(\theta, \tilde{x}\left(\theta, \alpha_{i}\right), \alpha_{i}\right)+\frac{F}{2}\right)\right) .
$$

\footnotetext{
31 Of course, this fixed cost must be large enough to ensure that two $\underline{\alpha}$-countries would not always build the project under complete information so that $(\mathrm{H} 2)$ holds.
} 
That definition already incorporates the fact that each government chooses pricing according to its preferences only and that the corresponding consumptions are $\tilde{x}\left(\theta, \alpha_{i}\right)$.

We can rewrite the countries' incentive constraints as:

$$
V_{i}(\bar{\alpha}) \geq V_{i}(\underline{\alpha})+\underset{\alpha_{-i}}{E}\left(p\left(\underline{\alpha}, \alpha_{-i}\right)\right)(\underset{\theta}{E}(\tilde{S}(\theta, \tilde{x}(\theta, \bar{\alpha}), \bar{\alpha})-\underset{\theta}{E}(\tilde{S}(\theta, \tilde{x}(\theta, \underline{\alpha}), \underline{\alpha})))
$$

and

$$
V_{i}(\underline{\alpha}) \geq V_{i}(\bar{\alpha})-E_{\alpha_{-i}}\left(p\left(\bar{\alpha}, \alpha_{-i}\right)\right)(\underset{\theta}{E}(\tilde{S}(\theta, \tilde{x}(\theta, \bar{\alpha}), \bar{\alpha})-\underset{\theta}{E}(\tilde{S}(\theta, \tilde{x}(\theta, \underline{\alpha}), \underline{\alpha}))) .
$$

Taking into account the countries' participation constraints, the IA's problem can now be written as:

$$
(\mathrm{TP}))_{\mathrm{L}}: \max _{\left\{p(\cdot), V_{i}(\cdot)\right\}} \sum_{i=1}^{2} \underset{\alpha_{i}}{E}\left(V_{i}\left(\alpha_{i}\right)\right)
$$

subject to Eqs. (8), (14), (15), (22), (23) and (24).

Of course, constrained efficiency may still be achieved even with the participation constraint (15) just as in the case where pricing can be fully controlled. To analyze more interesting cases characterized by some distortions, let us assume that the following condition holds:

$$
\begin{aligned}
& 2 q^{2} \underset{\theta}{E}(\tilde{S}(\theta, \tilde{x}(\theta, \bar{\alpha}), \bar{\alpha}))+2 q(1-q)(\underset{\theta}{E}(\tilde{S}(\theta, \tilde{x}(\theta, \bar{\alpha}), \bar{\alpha}))+\underset{\theta}{E}(\tilde{S}(\theta, \tilde{x}(\theta, \underline{\alpha}), \underline{\alpha}))) \\
& \quad<2 q^{2}(\underset{\theta}{E}(\tilde{S}(\theta, \tilde{x}(\theta, \bar{\alpha}), \bar{\alpha}))-\underset{\theta}{E}(\tilde{S}(\theta, \tilde{x}(\theta, \underline{\alpha}), \underline{\alpha}))) .
\end{aligned}
$$

Condition (25) is similar to Eq. (19) obtained when pricing can be fully controlled by the IA. Again, (H1) and (H2) ensure that the decision to build or not the project is casesensitive and depends on the concerns for redistribution of both countries.

Proposition 5. Assume that governments in each country keep control of pricing and that conditions (H1), (H2) and Eq. (25) hold. The optimal mechanism with voluntary participation of the countries is characterized as follows.

- The incentive constraint of a $\bar{\alpha}$-country (Eq. (23)) and the participation constraint of a $\underline{\alpha-o n e ~(E q . ~(15)) ~ a r e ~ b o t h ~ b i n d i n g . ~}$

- The decision to build the infrastructure is distorted with the project being realized less often than when countries' preferences are common knowledge. In particular, when countries are asymmetric, the probability of building the project is positive but always less than one: $\bar{p}_{L}=1, \hat{p}_{L} \in[0,1]$ and $p_{L}=0$.

- The constrained efficient levels of consumption $\tilde{x}\left(\theta, \alpha_{i}\right)$ are always chosen in both countries.

- By definition, the dichotomy between pricing and the decision to build or not the infrastructure holds. 
The IA can no longer play on prices to relax the rich country's incentive constraint and consumption is always constrained efficient. The only remaining screening tool available becomes the probability of making the project when countries are asymmetric, namely $\hat{p}$. As a result, we expect greater distortions in the decision to realize the project when pricing of the infrastructure is out of the IA's control. Cancelling more often the project becomes an imperfect substitute for the missing control on prices.

To prove this result, we have to compare two third-best policies and, as usual, this exercise is difficult because of the endogeneity of the multiplier. However, the next proposition confirms that the intuition above is true at least under some conditions.

Proposition 6. Assume that $\Delta \theta$ is small enough, then $\hat{p}_{L}<\hat{p}^{S B}$.

Alternatively, the setting described in this section can be viewed as resulting from an exogenous political constraint that forces the IA to let countries exert their sovereignty in choosing prices. Relinquishing these control rights may again lead to an inefficiently low provision of the infrastructure if pricing in both countries are kept independent.

\section{An IA with redistributive concerns}

So far, the IA was modeled as a benevolent maximizer of the sum of both countries' expected welfares just in line with Myerson and Sattherwaite (1983) approach to modeling a mediator in the bargaining process. The sole concern of this mediator was thus the expected (constrained) efficiency of the outcome. This characterizes the most favorable bargaining procedure from an ex ante viewpoint.

Let us now look at the optimal mechanism that would be chosen by an IA with a more active role on the redistribution side. Since bargaining will be less efficient, such a mediator with his own redistributive concerns could only be accepted by the countries if he subsidizes somewhat the project by bringing his own funds. ${ }^{32}$ Redistribution can be pursued at the aggregate level, i.e., between countries, or at the individuals level. Both cases are analyzed below.

\subsection{Redistribution across countries}

We now assume that the IA wants to maximize the following weighted sum of the aggregate welfare in both countries:

$$
\left.\sum_{i=1}^{2} \beta q V_{i}(\bar{\alpha})\right)+(1-\beta q) V_{i}(\underline{\alpha})=\sum_{i=1}^{2}\left(\underset{\alpha_{i}}{E}\left(V_{i}\left(\alpha_{i}\right)\right)-q(1-\beta)\left(V_{i}(\bar{\alpha})-V_{i}(\underline{\alpha})\right)\right)
$$

where $0<\beta<1$. This new objective function highlights the trade-off faced by the IA between looking for a (constrained) efficient outcome which maximizes the sum of

\footnotetext{
32 With that interpretation, the fixed cost $F$ should be understood as net of this subsidy.
} 
expected welfares in both countries and minimizing the costly welfare inequality that incentive compatibility at the countries level requires. ${ }^{33}$

Still assuming that countries can exert their sovereign rights and opt out of the mechanism if they wish so, the IA is constrained by the same budget-balanced, incentive and participation constraints than in Section 5. After consolidation of these constraints, the reduced form of the IA's problem writes as:

$$
\begin{gathered}
(\mathrm{TP})_{\mathrm{RC}} * \max _{\left\{p(\cdot), x_{i}(\cdot)\right\}} E\left\{p(\alpha)\left(\sum_{i=1}^{2} \underset{\theta}{E}\left(\tilde{S}\left(\theta, x_{i}(\theta, \alpha), \alpha_{i}\right)\right)\right)\right\} \\
-\sum_{i=1}^{2} v q(1-\beta) \Delta \alpha \Delta \theta{\underset{\alpha}{\alpha-i}}_{E}\left(p\left(\underline{\alpha}, \alpha_{-i}\right) v\left(x_{i}\left(\underline{\theta}, \underline{\alpha}, \alpha_{-i}\right)\right)\right)
\end{gathered}
$$

subject to Eq. (18).

Proposition 7. Assume that countries can opt out of the mechanism, that the IA has some redistributive concerns between countries and that conditions (H1), (H2) and Eq. (19) hold. The optimal mechanism is similar to that in Proposition 4. In particular, it entails:

- A distortion in the decision to build the infrastructure when countries have asymmetric preferences: $\bar{p}_{R C}=1, \hat{p}_{R C} \in[0,1], p_{R C}=0$.

- A downward distortion for the consumption of the poor in the $\underline{\alpha-c o u n t r y ;} x_{R C}(\bar{\theta}, \underline{\alpha}, \bar{\alpha})=$ $x^{*}(\bar{\theta})$ and $x_{R C}(\underline{\theta}, \underline{\alpha}, \bar{\alpha})<\tilde{x}(\underline{\theta}, \underline{\alpha})$ with

$$
\left(\underline{\theta}-\frac{v}{1-v}(1-\underline{\alpha}) \Delta \theta-\frac{(\lambda+1-\beta) v \Delta \alpha}{(1+\lambda)(1-v)(1-q)} \Delta \theta\right) v^{\prime}\left(x_{\mathrm{RC}}(\underline{\theta}, \underline{\alpha}, \bar{\alpha})\right)=c
$$

where $\lambda$ is the shadow cost of the feasibility constraint (18).

If the shadow cost $\lambda$ was the same for problems $(\mathrm{TP})^{\mathrm{SB} *}$ and $(\mathrm{TP})_{\mathrm{RC}} *$, the consumption would be more distorted when the IA has some redistributive concerns. The intuition is straightforward. The distribution of welfare across countries is now viewed as being directly costly and not, as in Section 5 , only indirectly because of the presence of the $\underline{\alpha}$-country's participation constraint. This new direct reason for reducing $V_{i}(\bar{\alpha})$ calls for extra downward distortions. ${ }^{34}$ Far from helping in improving expected welfare in the poor countries the redistributive concerns of the IA call for greater downward distortions in consumption and a reinforced link between pricing in both countries.

\footnotetext{
33 This objective can be rationalized in the same way as what we did for the objective functions of the governments themselves (see Appendix A for that case). By varying the reservation payoff of the poor countries, one describes several possible values of $\beta$.

34 A more formal comparison is made difficult by the fact that the shadow cost changes between the two problems and that computing explicitly those shadow costs is difficult as usual in second-best environments.
} 


\subsection{Redistribution within countries}

Let us look at the case where the IA has some concerns for poverty at the individual level and puts an extra exogenous positive weight $\mu$ on the utility levels of the poor agents. This reduced form can be rationalized by introducing explicitly a subsistence level (or reservation payoff) for the poorest agents into the constraints of a benevolent mediator. ${ }^{35}$ Formally, the IA now maximizes:

$$
\left(\sum_{i=1}^{2} \underset{\alpha_{i}}{E} V_{i}\left(\alpha_{i}\right)\right)+\mu\left(\sum_{i=1}^{2} \underset{\alpha}{E}\left(U_{i}(\underline{\theta}, \alpha)\right)\right)
$$

where $\mu>0$. Because the rich agents' incentive constraints within each country are binding, we have:

$$
\underset{\alpha_{-i}}{E}\left(U_{i}\left(\underline{\theta}, \alpha_{i}, \alpha_{-i}\right)\right)=V_{i}\left(\alpha_{i}\right)-\alpha_{i} v \Delta \theta \underset{\alpha_{-i}}{E}(p(\alpha) v(x(\underline{\theta}, \alpha))) .
$$

We can finally rewrite the IA's objective function as:

$$
\left.\underset{\alpha_{i}}{E}\left(V_{i}\left(\alpha_{i}\right)-\frac{\alpha_{i} \mu \nu}{1+\mu} \Delta \theta \underset{\alpha_{-i}}{E}(p(\alpha) v(x \underline{\theta}, \alpha))\right)\right)
$$

Because $\mu>0$, the welfare inequality within countries is viewed as costly by the IA.

Following the same steps as before, the reduced form of the IA's problem can be written as:

$$
\left.(\mathrm{TP})_{\mathrm{RA}}^{*}: \max _{\left\{p(\cdot), x_{i}(\cdot)\right\}} E\left\{p(\alpha)\left(\sum_{i=1}^{2} E\left(\tilde{\theta}(\theta), x_{i}(\theta, \alpha), \alpha_{i}\right)\right)-\frac{\mu}{1+\mu} v \alpha_{i} \Delta \theta v\left(x_{i}(\underline{\theta}, \alpha)\right)\right)\right)
$$

subject to Eq. (18).

We need to describe a pair of conditions similar to (H1) and (H2) which ensure that the project would be done under complete information on the preferences profile if and only if at least one of the country is a rich one. Those conditions are:

$$
\begin{aligned}
& \underset{\theta}{E}\left(\tilde{S}\left(\theta, \tilde{x}\left(\theta, \frac{\bar{\alpha}}{1+\mu}\right), \frac{\bar{\alpha}}{1+\mu}\right)\right)+\underset{\theta}{E}\left(\tilde{S}\left(\theta, \tilde{x}\left(\theta, \frac{\underline{\alpha}}{1+\mu}\right), \frac{\underline{\alpha}}{1+\mu}\right)\right)>0, \\
& \underset{\theta}{E}\left(\tilde{S}\left(\theta, \tilde{x}\left(\theta, \frac{\underline{\alpha}}{1+\mu}\right), \frac{\underline{\alpha}}{1+\mu}\right)\right)<0 .
\end{aligned}
$$

\footnotetext{
35 Because of incentive compatibility within countries, the same subsistence level is also satisfied for the rich agents.
} 
Proposition 8. Assume that countries can opt out of the mechanism, that the IA has some redistributive concerns and cares about poverty within countries and that conditions (H1'), (H2') and Eq. (19) hold. The optimal mechanism is similar to that in Proposition 4. In particular, it entails:

- A distortion in the decision to build the infrastructure when countries have asymmetric preferences: $\bar{p}_{R A}=1, \hat{p}_{R A} \in[0,1], p_{R A}=0$.

- Strong downward distortions for the consumption of the poor in both a $\bar{\alpha}$ - and $a \underline{\alpha}-$ country if the project is realized:

$$
\begin{aligned}
& \left(\underline{\theta}-\frac{v}{1-v}\left(1-\frac{\bar{\alpha}}{1+\mu}\right) \Delta \theta\right) v^{\prime}\left(x_{\mathrm{RA}}(\underline{\theta}, \bar{\alpha}, \bar{\alpha})\right)=c \\
& \left(\underline{\theta}-\frac{v}{1-v}\left(1-\frac{\underline{\alpha}}{1+\mu}\right) \Delta \theta-\frac{\lambda v q \Delta \alpha}{(1+\lambda)(1-v)(1-q)} \Delta \theta\right) v^{\prime}\left(x_{\mathrm{RA}}(\underline{\theta}, \underline{\alpha}, \bar{\alpha})\right)=c
\end{aligned}
$$

where $\lambda$ is the shadow cost of the feasibility constraint (18).

The IA's concern for poverty limits the prices charged to the poor in both countries. The bulk of the contribution is thus borne by the rich agents. This hardens their incentive constraint and requires further distortions of the consumption of the poor to make their allocation less attractive to the rich. As a result, there will be strong distortions on consumptions even if both countries are rich.

Looking at the solution, everything happens as if, a priori, the preferences for redistribution could be characterized by a new parameter $\beta=(\alpha / 1+\mu)<\alpha$ and then an analysis similar to that of Section 5 follows. The IA's concerns for poverty trickles down again to the local level. The external mechanism for public good provision puts enough constraints on local governments to modify their preferences for redistribution and makes them behave as being more averse to inequality than what they really are.

\section{Extensions and conclusion}

In this paper, we have shown how asymmetric information may impede efficiency in the provision of transnational projects. Optimal mechanisms may call for cancelling more often the projects than when the preference profile for redistribution of the countries involved is common knowledge. Consumption by the poor is also reduced for incentive compatibility reasons and prices in both countries are linked.

Transnational projects have also an impact on the distribution of utilities within countries. The governments' concerns for redistribution might be exacerbated by the external constraints imposed by the IA's mechanism. The IA's concerns for redistribution either across or within countries spill over to the local level, reinforce the governments' own concerns for redistribution, affects pricing and the decision to build or not the infrastructure. 
Another common theme of the models developed above is that the number of instruments for enforcing the mechanism determines the size of the inefficiency. As the IA has less control of the project (either because it cannot force acceptance by sovereign countries or because it cannot control prices), inefficiency increases and the project is less likely to be implemented. At a broad level, these results suggest that some form of sovereignty loss is needed to facilitate the structural investments which are necessary for growth. Otherwise, investments may be kept inefficiently low to preserve ex post agreement of countries.

Various extensions of our framework would be worth to be undertaken.

\subsection{Comparison with other forms of financing}

A more complete analysis should compare the costs and benefits of various institutions for accessing to those infrastructures. In this respect, it is striking that the very argument of sovereignty loss that was used to criticize financing by private foreign investors comes with a revenge in the case of an a priori more cooperative bargaining solution. More generally, it would be worth to compare the outcome achieved with those collective mechanisms with what is achieved when countries decide to get access to those infrastructures by using foreign private investors. One important question from a policy point of view is to know whether the cooperative solution discussed in this paper is subject to as much renegotiation as the traditional devices. Even though no theory is yet available, some comments can already been made. Indeed, it is well known from adverse selection models that transaction costs of contracting under asymmetric information are lower when bargaining powers are more equal than with asymmetric bargaining powers. The strong bargaining position of foreign private financiers in contracting suggests therefore that there exist then large costs of signing and renegotiating contracts that could be (at least partially) avoided through joint effort by countries.

\subsection{Political economy}

Our approach so far has been mostly normative and an obvious extension would be to deal more precisely with the political economy side of the model. Political economy considerations could help to endogenize the preferences for redistribution at the local level. An important issue that could be analyzed is the corruption of governments, a phenomenon which is very likely to arise given the important financial stakes involved with transnational projects. Politics could also help to understand the IA's objective function if the latter was modeled as a more active actor obeying to his own incentives and reputational concerns.

\subsection{Voluntary contributions}

In our framework, we gave to the IA a strong commitment ability by having it move first and commit itself to a mechanism. This approach yields an upper bound on what can be achieved through any bargaining mechanism between countries. An alternative and weaker view of the IA would be to see it as simply collecting voluntary contributions. Although there exists now a literature on voluntary contributions under asymmetric 
information, ${ }^{36}$ none of these papers have considered the case where voluntary contributions are made by countries and not by individuals. It would be worth to provide such an extension and compare the equilibrium outcomes with that achieved under the more centralized mechanism described in this paper.

\subsection{Externalities and scale economies}

The externality between countries was modeled in a rather crude way. In our model, scale economies can only be achieved by building a common infrastructure. Other forms of increasing returns could be considered. For instance, it may become at the margin easier to provide consumption to new consumers as others are already served. ${ }^{37}$ Decreasing marginal costs introduce some new features. Downward distortions in consumption must be somewhat mitigated to keep low marginal costs. Inducing such distortions as screening devices seems less useful than playing on the probabilities of building the project. One may expect that the infrastructure should be less often built as increasing returns become more important, whereas at the same time consumption should not bet distorted too much.

Network externality may also affect demand. ${ }^{38}$ The existence of such a transnational network externality gives a new role to the IA who now proposes prices which make each country internalize the impact of its own consumption choices on the other. Of course, this requires that the IA can fully control those prices. In the absence of such a control, local governments would choose prices non-cooperatively and this would lead to inefficient consumption because of a (non-internalized) positive externality. However, to benefit from the network externality, consumptions should not be too distorted. Again, the only screening tool available to the IA remains then the probability of cancelling the project.

\subsection{Local infrastructures}

An alternative to the transnational project may be to build infrastructures of a lesser scale in each country. The reservation payoff of each country is no longer zero and depends explicitly on its preferences for redistribution. New issues in the design of the collective agreement may appear. Ex post agreement between the countries may now become harder and as a result one should expect the project to be less often realized. On the other hand, one important lesson of adverse selection models with type-dependent reservation payoffs is that those outside opportunities, when binding, tend to reduce allocative inefficiencies and consumption should be kept close to the second-best. ${ }^{39}$

\subsection{Global public goods}

Even though we had in mind specific examples of transnational infrastructures for developing countries in writing this paper, its lessons may have also some value to

\footnotetext{
36 See Menezes et al. (2001), Laussel and Palfrey (2003) and Martimort and Moreira (2003) for instance.

37 A typical example could be irrigation.

38 For instance, in the case of a telecommunications network, our assumption that the benefits of consuming in one country do not depend on the consumption in another nearby country may seem unrealistic.

39 See Laffont and Martimort (2002, Chap. 3).
} 
understand the governance of more general global public goods (or bads) like global warming, disease prevention, trade agreements, etc.

We hope to investigate some of the specific issues raised by those public goods in further research.

\section{Acknowledgements}

We thank Paulina Beato for suggesting this research and seminar participants in Toulouse, especially Pierre Dubois, for very useful comments on an earlier version. Two referees made insightful comments that have much improved the paper. The usual disclaimer applies. After this paper was completed but before it was published, JeanJacques Laffont passed away. We will miss him.

\section{Appendix A. Endogenizing government's preferences and asymmetric information}

Let us suppose that pricing is used to cover a random deficit $\tilde{\kappa} \in\{\underline{\kappa}, \bar{\kappa}\}$ with respective probabilities $q$ and $1-q$ and $\bar{\kappa}>\underline{\kappa}$. We suppose that the government is benevolent and maximizes the sum of utilities of the different types of agents subject to the agents' incentive and participation constraints that we will normalize at some exogenous level $U_{0}$. Focusing (as usual) only on the rich agent's incentive constraint and the poor agent's participation one, the government's problem can be written as:

$$
\max _{\{x(\cdot), U(\cdot)\}} v U(\bar{\theta})+(1-v) U(\underline{\theta}) .
$$

subject to

$$
\begin{aligned}
& U(\bar{\theta})-U(\underline{\theta}) \geq \Delta \theta v(x(\underline{\theta})), \\
& U(\underline{\theta}) \geq U_{0},
\end{aligned}
$$

and

$$
v U(\bar{\theta})+(1-v) U(\underline{\theta})+\kappa \leq v(\bar{\theta} v(x(\bar{\theta}))-c x(\bar{\theta}))+(1-v)(\underline{\theta} v(x(\underline{\theta}))-c x(\underline{\theta})),
$$

where the latter constraint is the budget constraint of the state when the deficit is $\kappa$. Of course, this constraint is binding at the optimum.

From Eqs. (A.1) and (A.2), Eq. (A.3) implies:

$$
v(\bar{\theta} v(x(\bar{\theta}))-c x(\bar{\theta}))+(1-v)(\underline{\theta} v(x(\underline{\theta}))-c x(\underline{\theta})) \geq \kappa+v \Delta \theta v(x(\underline{\theta}))+U_{0} .
$$

When $\kappa$ is large enough (but not too large so that the constrained set remains non-empty), this constraint is clearly no longer satisfied by the first-best optimal levels of consumptions 
$x^{*}(\underline{\theta})$ and $x^{*}(\bar{\theta})$. Then, Eq. (A.4) is binding at the optimum (and consequently Eqs. (A.1) and (A.2) are also binding). We can rewrite the government's problem as

$$
\left.\max _{\{x(\cdot)\}} v(\bar{\theta} v(x(\bar{\theta}))-c x(\bar{\theta}))+(1-v)(\underline{\theta} v)(x(\underline{\theta}))-c x(\underline{\theta})\right)
$$

subject to Eq. (A.4).

Denoting by $\mu(\kappa)$ the positive multiplier of Eq. (A.4), the government maximizes

$$
(1-v)(\underline{\theta} v(x(\underline{\theta}))-c x(\underline{\theta}))+v(\bar{\theta} v(x(\bar{\theta}))-c x(\bar{\theta}))-\frac{v \mu(\kappa)}{1+\mu(\kappa)} \Delta \theta v(x(\underline{\theta}))
$$

for some $\mu(\kappa)>0$, where $\mu(\kappa)$ is given by the slackness condition. Of course, $\mu(\kappa)$ is increasing in $\kappa$. Denoting $1-\bar{\alpha}=\mu(\bar{\kappa}) /(1+\mu(\underline{\kappa}))$ and $1-\underline{\alpha}=\mu(\underline{\kappa}) /(1+\mu(\underline{\kappa}))$, we observe that everything happens as if the government maximizes an objective function of the type $\alpha v U(\bar{\theta})+(1-\alpha v) U(\underline{\theta})$. Private information on the parameter $\alpha$ can thus be viewed as a reduced form for private information on the shock $\kappa$ hitting the budget constraint of the state.

\section{Appendix B. (Transformation of (TP)FB into (TP)FB* and Proof of Proposition 1}

First, we rewrite the objective function of the IA which becomes:

$$
\sum_{i=1}^{2}\left(v U_{i}(\bar{\theta}, \alpha)+(1-v) U_{i}(\underline{\theta}, \alpha)-v\left(1-\alpha_{i}\right)\left(U_{i}(\bar{\theta}, \alpha)-U_{i}(\underline{\theta}, \alpha)\right)\right)
$$

for a given preferences profile $\alpha=\left(\alpha_{1}, \alpha_{2}\right)$. Clearly, this shows that Eq. (A.4) must be binding at the optimum and we get thus:

$$
\left.\sum_{i=1}^{2} \tilde{V}_{i}(\alpha)=p(\alpha)\left\{\sum_{i=1}^{2} \underset{\theta}{E}\left(S\left(\theta, x_{i}(\theta, \alpha)\right)\right)\right\}-\sum_{i=1}^{2} v\left(1-\alpha_{i}\right)\left(U_{i}(\bar{\theta}, \alpha)-U_{i}(\underline{\theta}, \alpha)\right)\right)
$$

Since $\alpha_{i}<1$, the second term on the right-hand side of Eq. (A.5) is minimized when Eq. (6) is binding. Note then that Eq. (7) is slack as soon as $x_{i}(\underline{\theta}, \alpha)<x_{i}(\bar{\theta}, \alpha)$, a monotonicity condition that will be checked ex post.

Inserting Eq. (6) binding into Eq. (A.5) yields the maximand of (TP) ${ }^{\mathrm{FB}}$, namely:

$$
p(\alpha)\left\{\sum_{i=1}^{2} \underset{\theta}{E}\left(\tilde{S}\left(\theta, x_{i}(\theta, \alpha), \alpha_{i}\right)\right)\right\} \text {. }
$$

The second-best consumptions $\tilde{x}\left(\theta, \alpha_{i}\right)$ maximize this expression. Of course, $\tilde{x}\left(\underline{\theta}, \alpha_{i}\right)$ $<x^{*}(\bar{\theta})$ and Eq. (6) binding implies that Eq. (7) is slack. 
Under assumptions (H1) and (H2), we have $\hat{p}^{*}=1$ and $p^{*}=0$. Moreover, by definition of $\tilde{x}(\theta, \alpha)$ and the fact that the government in the poor country is more averse to inequality that in the rich country, $\tilde{x}(\underline{\theta}, \underline{\alpha})<\tilde{x}(\underline{\theta}, \bar{\alpha})$. Finally, $\underset{\theta}{E}(\tilde{S}(\theta, \tilde{x}(\theta, \bar{\alpha}), \bar{\alpha}))>\underset{\theta}{E}(\tilde{S}(\theta, \tilde{x}(\theta, \underline{\alpha}), \underline{\alpha}))$. Hence, assumption (H1) implies also that $\bar{p}^{*}=1{ }^{\theta}$

\section{Appendix C. Transformation of (TP) $)_{0}$ into (TP)\% and Proof of Proposition 2}

First, we observe that:

$$
\begin{aligned}
V_{i}\left(\alpha_{i}\right)= & \underset{\alpha_{-i}}{E}\left(\underset{\theta}{E}\left(U_{i}(\theta, \alpha)\right)-v\left(1-\alpha_{i}\right)\left(U_{i}(\bar{\theta}, \alpha)-U_{i}(\underline{\theta}, \alpha)\right)\right) \\
= & \underset{\alpha_{-1}}{E}\left(p(\alpha)\left(\underset{\theta}{E}\left(S(\theta, x(\theta, \alpha))+\frac{F}{2}\right)-v\left(1-\alpha_{i}\right)\left(U_{i}(\bar{\theta}, \alpha)-U_{i}(\underline{\theta}, \alpha)\right)\right)\right. \\
& -\underset{\alpha_{-i}}{E}\left(T_{i}(\alpha)\right) .
\end{aligned}
$$

For a symmetric mechanism, $\underset{\alpha}{E}\left(T_{i}\left(\bar{\alpha}, \alpha_{-i}\right)=q \bar{T}+(1-q) \hat{T}_{1}\right.$ and $\underset{\alpha}{E}\left(T_{i}\left(\underline{\alpha}, \alpha_{-i}\right)=q \hat{T}_{2}+\right.$ $(1-q) \underline{T}$. Hence, still using the symmetry of the mechanism, we have:

$$
\begin{aligned}
\sum_{i=1}^{2} \underset{\alpha_{i}}{E}\left(V_{i}\left(\alpha_{i}\right)\right)= & E\left(p(\alpha)\left(\left(\sum_{i=1}^{2} \underset{\theta}{E}\left(S\left(\theta, x_{i}(\theta, \alpha)\right)\right)+F\right)\right)\right. \\
& -\sum_{i=1}^{2} v\left(1-\alpha_{i}\right)\left(U_{i}(\bar{\theta}, \alpha)-U_{i}(\underline{\theta}, \alpha)\right)-E_{\alpha}\left(\sum_{i=1}^{2} T_{i}(\alpha)\right) .
\end{aligned}
$$

Maximization of this expression subject to the ex post budget constraints (8) is obtained when all such constraints are binding. Moreover, as in the Proof of Proposition 1 the right-hand side above is maximized when Eq. (6) is binding, i.e.,

$$
U_{i}(\bar{\theta}, \alpha)-U_{i}(\underline{\theta}, \alpha)=p(\alpha) \Delta \theta v\left(x_{i}(\underline{\theta}, \alpha)\right), \text { for all } i \in\{1,2\} \text {. }
$$

Again, the fact that $x_{i}(\underline{\theta}, \alpha)<x_{i}(\bar{\theta}, \alpha)$ for the solution ensures that Eq. (7) is then slack.

Gathering all those facts, we obtain:

$$
\sum_{i=1}^{2} \underset{\alpha_{i}}{E}\left(V_{i}\left(\alpha_{i}\right)\right)=E_{\alpha}\left\{p(\alpha)\left(\sum_{i=1}^{2} \underset{\theta}{E}\left(\tilde{S}\left(\theta, x_{i}(\theta, \alpha), \alpha_{i}\right)\right)\right)\right\}
$$

i.e., the maximand of $\left(\mathrm{TP}_{0}\right)^{*}$ with the incentive constraints of both countries (Eqs. (11) and (12)). There exists a whole range of solutions to this problem which are all characterized by the same consumptions and the same aggregate welfare. We will focus on the symmetric ones and thus omit indices. This allows us a clear characterization of the incentive compatible pairs $(V(\bar{\alpha}), V(\underline{\alpha}))$. There is still a whole 
range of such symmetric pairs $(V(\bar{\alpha}), V(\underline{\alpha}))$ which satisfy Eq. (A.6) as an equality and the incentive constraints (11) and (12).

Since Eq. (A.6) holds as an equality, defining $V(\underline{\alpha})$ defines also $V(\bar{\alpha})$. All possible values of $V(\underline{\alpha})$ describe the interval $\left[V_{\mathrm{m}}(\underline{\alpha}), V_{\mathrm{M}}(\underline{\alpha})\right]$ where:

$$
\begin{aligned}
& V_{\mathrm{m}}(\underline{\alpha})=\frac{A}{2}-v q \Delta \alpha \Delta \theta v(\tilde{x}(\underline{\theta}, \bar{\alpha})), \\
& V_{\mathrm{M}}(\underline{\alpha})=\frac{A}{2}-v q^{2} \Delta \alpha \Delta \theta v(\tilde{x}(\underline{\theta}, \underline{\alpha})) ;
\end{aligned}
$$

and $A$ is the right-hand side of Eq. (A.6) computed for the constrained efficient probabilities $\bar{p}^{*}=\hat{p}^{*}=1, p^{*}=0$ and the second-best consumptions $\tilde{x}(\theta, \alpha)$. We have thus:

$$
A=2 q^{2} \underset{\theta}{E}(\tilde{S}(\theta, \tilde{x}(\theta, \bar{\alpha}), \bar{\alpha}))+2 q(1-q)(\underset{\theta}{E}(\tilde{S}(\theta, \tilde{x}(\theta, \bar{\alpha}), \bar{\alpha}))+\underset{\theta}{E}(\tilde{S}(\theta, \tilde{x}(\theta, \underline{\alpha}), \underline{\alpha}))) .
$$

Since $q<1$ and $\tilde{x}(\underline{\theta}, \underline{\alpha})<\tilde{x}(\underline{\theta}, \bar{\alpha})$, we have indeed $V_{\mathrm{M}}(\underline{\alpha})>V_{\mathrm{m}}(\underline{\alpha})$. For any $V(\underline{\alpha})$ in $\left[V_{\mathrm{m}}(\underline{\alpha}), V_{\mathrm{M}}(\underline{\alpha})\right]$ and the corresponding value of $V(\bar{\alpha})$ obtained when Eq. (A.6) is binding, we can find the values of the symmetric transfers $\left(\bar{T}, \hat{T}_{1}, \hat{T}_{2}, \underline{T}\right)$, which implement these utility levels as solutions to the following system:

$$
\begin{aligned}
& V(\underline{\alpha})= q \hat{p}^{*}\left(\underset{\theta}{E}\left(\tilde{S}\left(\theta, x^{\mathrm{SB}}(\theta, \underline{\alpha}, \bar{\alpha})\right)\right)+\frac{F}{2}\right)-\left(q \hat{T}_{2}+(1-q) \underline{T}\right) \\
& V(\bar{\alpha})= q \bar{p}^{*}\left(\underset{\theta}{E}\left(\tilde{S}\left(\theta, x^{\mathrm{SB}}(\theta, \bar{\alpha}, \bar{\alpha})\right)\right)+\frac{F}{2}\right)+(1-q) \hat{p}^{*}\left(\underset{\theta}{E}\left(\tilde{S}\left(\theta, x^{\mathrm{SB}}(\theta, \bar{\alpha}, \underline{\alpha})\right)\right)+\frac{F}{2}\right) \\
&-\left(q \bar{T}+(1-q) \hat{T}_{1}\right) \\
& 2 \bar{T}= \bar{p}^{*} F \\
& \hat{T}_{1}+ \hat{T}_{2}=\hat{p}^{*} F, \\
& 2 \underline{T}=\underline{p}^{*} F,
\end{aligned}
$$

where $\bar{p}^{*}=\hat{p}^{*}=1$ and $p^{*}=0$.

Note that Eqs. (A.9) and (A.11) yield immediately $\bar{T}=F / 2$ and $T=0$ meaning that no transfer is paid when the project is not done. Finally, $\left(\hat{T}_{1}, \hat{T}_{2}\right)$ is immediately obtained as a solution to Eqs. (A.7) and (A.10). 


\section{Appendix D. Proof of Proposition 3}

The same decision rule and consumptions as in Proposition 2 can no longer be obtained when the preferences for redistribution are unknown if $V_{M}(\underline{\alpha})<0$. Writing this condition yields Eq. (16).

\section{Appendix E. Transformation of (TP) ${ }^{\mathrm{SB}}$ into (TP) ${ }^{\mathrm{SB} *}$ and Proof of Proposition 4}

Since Eq. (16) holds, Eq. (17) is not satisfied by the solution obtained when the preferences profile $\alpha=\left(\alpha_{1}, \alpha_{2}\right)$ is common knowledge. Hence, we should look for a solution of (TP) ${ }^{\mathrm{SB}}$ such that Eqs. (11) and (15) (and thus Eq. (18)) will be binding. The fact that Eqs. (11) and (15) are satisfied implies (for a positive $x(\underline{\theta}, \underline{\alpha}, \bar{\alpha})$ ) that Eq. (14) holds strictly, so that this constraint can be omitted in the optimization below.

Let us omit also the incentive constraint (12) (which can be checked ex post). Then, we can rewrite $(\mathrm{TP})^{\mathrm{SB}}$ in a more compact way as:

$$
\begin{aligned}
& (\mathrm{TP})^{\mathrm{SB}}: \max _{\left\{p(\cdot), x_{i}(\cdot), V_{i}(\cdot)\right\}} E\left\{p(\alpha)\left(\sum_{i=1}^{2} \underset{\theta}{E}\left(S\left(\theta, x_{i}(\theta, \alpha), \alpha_{i}\right)\right)\right)\right. \\
& \left.-\sum_{i=1}^{2} v\left(1-\alpha_{i}\right)\left(U_{i}(\bar{\theta}, \alpha)-U_{i}(\underline{\theta}, \alpha)\right)\right\},
\end{aligned}
$$

subject to (Eqs. (6), (7), (11) and (15) and

$$
\begin{gathered}
\sum_{i=1}^{2} \underset{\alpha_{i}}{E}\left(V_{i}\left(\alpha_{i}\right)\right)=\underset{\alpha}{E}\left\{p(\alpha)\left(\sum_{i=1}^{2} \underset{\theta}{E}\left(S\left(\theta, x_{i}(\theta, \alpha), \alpha_{i}\right)\right)\right)\right. \\
\left.-\sum_{i=1}^{2} v\left(1-\alpha_{i}\right)\left(U_{i}(\bar{\theta}, \alpha)-U_{i}(\underline{\theta}, \alpha)\right)\right\} .
\end{gathered}
$$

Using Eqs. (11) and (15), we get

$$
\begin{gathered}
\underset{\alpha}{E}\left\{p(\alpha)\left(\sum_{i=1}^{2} \underset{\theta}{E}\left(S\left(\theta, x_{i}(\theta, \alpha), \alpha_{i}\right)\right)\right)-\sum_{i=1}^{2} v\left(1-\alpha_{i}\right)\left(U_{i}(\bar{\theta}, \alpha)-U_{i}(\underline{\theta}, \alpha)\right)\right\} \\
=\sum_{i=1}^{2} \underset{\alpha_{i}}{E}\left(V_{i}\left(\alpha_{i}\right)\right) \geq \sum_{i=1}^{2} v q \Delta \alpha \underset{\alpha_{-i}}{E}\left(U_{i}\left(\bar{\theta}, \underline{\alpha}, \alpha_{-i}\right)-U_{i}\left(\underline{\theta}, \underline{\alpha}, \alpha_{-i}\right)\right) .
\end{gathered}
$$

Optimizing first with respect to $U_{i}($.$) , Eq. (6) is binding to increase the maximand in$ $(\mathrm{TP})^{\mathrm{SB}^{\prime}}$ and relax constraint Eq. (A.13). Eq. (7) is slack as it can be checked ex post. 
Inserting the corresponding value of $U_{i}(\bar{\theta}, \alpha)-U_{i}(\underline{\theta}, \alpha)$ into Eq. (A.13) and the maximand of $(\mathrm{TP})^{\mathrm{SB}^{\prime}}$, we get Eq. (18) and the expression of the maximand of IA's problem as $(\mathrm{TP})^{\mathrm{SB} *}$.

Let us denote by $\lambda$ the positive multiplier of Eq. (18) into $(\mathrm{TP})^{\mathrm{SB} *}$. The Lagrangean is:

$$
\underset{\alpha}{E}\left\{(1+\lambda) p(\alpha)\left(\sum_{i=1}^{2} \underset{\theta}{E}\left(\tilde{S}\left(\theta, x_{i}(\theta, \alpha), \alpha_{i}\right)\right)\right)\right\}-\sum_{i=1}^{2} \lambda v q \Delta \alpha \Delta \theta \underset{\alpha_{-i}}{E}\left(v\left(x_{i}\left(\underline{\theta}, \underline{\alpha}, \alpha_{-i}\right)\right)\right) .
$$

Optimizing with respect to $x_{i}($.$) yields a symmetric solution such that$

- For a rich country,

$\bar{\theta} v^{\prime}\left(x^{\mathrm{SB}}\left(\bar{\theta}, \bar{\alpha}, \alpha_{-i}\right)\right)=c, \quad \forall \alpha_{-i}$

and so $x^{\mathrm{SB}}\left(\bar{\theta}, \bar{\alpha}, \alpha_{-i}\right)=x^{*}(\bar{\theta})$;

$\left(\underline{\theta}-\frac{v(1-\bar{\alpha})}{1-v} \Delta \theta\right) v^{\prime}\left(x^{\mathrm{SB}}\left(\underline{\theta}, \bar{\alpha}, \alpha_{-i}\right)\right)=c, \quad \forall \alpha_{-i}$

and so $x^{\mathrm{SB}}\left(\underline{\theta}, \bar{\alpha}, \alpha_{-i}\right)=\tilde{x}(\underline{\theta}, \bar{\alpha})$;

- For a poor country,

$\bar{\theta} v^{\prime}\left(x^{\mathrm{SB}}\left(\bar{\theta}, \underline{\alpha}, \alpha_{-i}\right)\right)=c, \quad \forall \alpha_{-i}$

and so $x^{\mathrm{SB}}\left(\bar{\theta}, \underline{\alpha}, \alpha_{-i}\right)=x^{*}(\bar{\theta})$;

$\left(\underline{\theta}-\frac{v(1-\underline{\alpha})}{1-v} \Delta \theta-\frac{\lambda q v \Delta \alpha}{(1+\lambda)(1-q)(1-v)} \Delta \theta\right) v^{\prime}\left(x^{\mathrm{SB}}\left(\underline{\theta}, \underline{\alpha}, \alpha_{-i}\right)\right)=c, \quad \forall \alpha_{-i}$

and so $x^{\mathrm{SB}}\left(\underline{\theta}, \underline{\alpha}, \alpha_{-i}\right)<\tilde{x}(\underline{\theta}, \underline{\alpha})$ since $\lambda>0$.

Let us now optimize with respect to $\bar{p}, \hat{p}$ and $\underline{p}$. We obtain:

$$
\bar{p}^{\mathrm{SB}}=1 \Leftrightarrow 2(1+\lambda) q^{2} \underset{\theta}{E}(\tilde{S}(\theta, \tilde{x}(\theta, \bar{\alpha}), \bar{\alpha}))>0
$$

which holds from (H1),

$$
\begin{aligned}
\hat{p}^{\mathrm{SB}} & \in[0,1] \Leftrightarrow 2(1+\lambda) q(1-q)\left(\underset{\theta}{E}\left(\tilde{S}(\theta, \tilde{x}(\theta, \bar{\alpha}, \bar{\alpha}))+\underset{\theta}{E}\left(\tilde{S}\left(\theta, x^{\mathrm{SB}}(\theta, \underline{\alpha}, \bar{\alpha}), \underline{\alpha}\right)\right)\right)\right. \\
& =2 \lambda q^{2} v \Delta \alpha \Delta \theta v\left(x^{\mathrm{SB}}(\underline{\theta}, \underline{\alpha}, \bar{\alpha})\right), \\
\underline{p}^{\mathrm{SB}} & =0 \Leftrightarrow 2(1+\lambda)(1-q)^{2} \underset{\theta}{E}\left(\tilde{S}\left(\theta, x^{\mathrm{SB}}(\theta, \underline{\alpha}, \underline{\alpha}), \underline{\alpha}\right)\right) \\
& <2 \lambda q(1-q) v \Delta \alpha \Delta \theta v\left(x^{\mathrm{SB}}(\underline{\theta}, \underline{\alpha}, \underline{\alpha})\right) .
\end{aligned}
$$


This latter inequality holds since $x^{\mathrm{SB}}(\underline{\theta}, \underline{\alpha}, \underline{\alpha})<\tilde{x}(\underline{\theta}, \underline{\alpha})$ implies that $\underset{\theta}{E}\left(\tilde{S}\left(\theta, x^{\mathrm{SB}}(\theta, \underline{\alpha}, \underline{\alpha})\right.\right.$, $\underline{\alpha}))<\underset{\theta}{E}(\tilde{S}(\theta, \tilde{x}(\theta, \underline{\alpha}), \underline{\alpha}))<0$ from condition $(\mathrm{H} 2)$.

From Eq. (18) binding, and taking also into account that $\bar{p}^{\mathrm{SB}}=1$ and $\underline{p}^{\mathrm{SB}}=0$, we get that $\hat{p}^{\mathrm{SB}}$, if it belongs to $[0,1]$, is the solution to the following equation:

$$
\begin{aligned}
& \hat{p}^{\mathrm{SB}}(1-q)\left(\underset{\theta}{E}(\tilde{S}(\theta, \tilde{x}(\theta, \bar{\alpha}), \bar{\alpha}))+\underset{\theta}{E}\left(\tilde{S}\left(\theta, x^{\mathrm{SB}}(\theta, \underline{\alpha}, \bar{\alpha}), \underline{\alpha}\right)\right)\right)+\underset{\theta}{\operatorname{E}}(\tilde{S}(\theta, \tilde{x}(\theta, \bar{\alpha}), \bar{\alpha})) \\
& \quad=q v \hat{p}^{\mathrm{SB}} \Delta \alpha \Delta \theta v\left(x^{\mathrm{SB}}(\underline{\theta}, \underline{\alpha}, \bar{\alpha})\right) .
\end{aligned}
$$

Using Eqs. (A.15) and (A.17), we obtain:

$$
\hat{p}^{\mathrm{SB}} \frac{v}{1+\lambda} \Delta \alpha \Delta \theta v\left(x^{\mathrm{SB}}(\underline{\theta}, \underline{\alpha}, \bar{\alpha})\right)=\underset{\theta}{E}(\tilde{S}(\theta, \tilde{x}(\theta, \bar{\alpha}), \bar{\alpha}))
$$

The right-hand side is positive by condition (H1). Hence, $\hat{p}^{\mathrm{SB}}>0$. Let us now show that $\hat{p}^{\mathrm{SB}}<1$ is necessary when condition (19) holds. Observe that $x_{\infty}(\underline{\theta}, \underline{\alpha})$ defined by Eq. (20) maximizes $-q v \Delta \alpha \Delta \theta v(x)+(1-q)(1-v) \tilde{S}(\underline{\theta}, x, \underline{\alpha})$. Therefore, even the strongest possible distortion on $x(\underline{\theta}, \underline{\alpha}, \bar{\alpha})$ makes it impossible to satisfy Eq. (18). A distortion of $\hat{p}$ is necessary.

Finally, to find the values of the transfers $\left(\bar{T}, \hat{T}_{1}, \hat{T}_{2}, \underline{T}\right)$ one can proceed as in the Proof of Proposition 3. Note that there is no freedom in the choice of $V(\underline{\alpha})$ which is always zero.

\section{Appendix F. Proof of Proposition 5}

When (H1) and (H2) hold, the optimal policy when the preferences profile $\alpha=\left(\alpha_{1}, \alpha_{2}\right)$ is common knowledge is thus $\bar{p}^{*}=\hat{p}^{*}=1, p^{*}=0$.

We now transform $(\mathrm{TP})_{\mathrm{L}}$ into $(\mathrm{TP})_{\mathrm{L}}^{*} \bar{b}$ elow:

$$
(\mathrm{TP})_{\mathrm{L}}^{*}: \max _{\{p(\cdot)\}} E\left\{p(\alpha)\left(\underset{\theta}{E}\left(\sum_{i=1}^{2}\left(\tilde{S}\left(\theta, \tilde{x}\left(\theta, \alpha_{i}\right), \alpha_{i}\right)\right)\right)\right\},\right.
$$

subject to

$$
\begin{aligned}
& \underset{\alpha}{E}\left\{p(\alpha)\left(\underset{\theta}{E}\left(\sum_{i=1}^{2} \tilde{S}\left(\theta, \tilde{x}\left(\theta, \alpha_{i}\right), \alpha_{i}\right)\right)\right\}-\sum_{i=1}^{2} q \underset{\alpha_{i}}{E}\left(p\left(\underline{\alpha}, \alpha_{-i}\right)\right)(\underset{\theta}{E}(\tilde{S}(\theta, \tilde{x}(\theta, \bar{\alpha}), \bar{\alpha}))\right. \\
& \quad-\underset{\theta}{E}(\tilde{S}(\theta, \tilde{x}(\theta, \underline{\alpha}), \underline{\alpha}))) \geq 0 .
\end{aligned}
$$


To do so, we proceed as before and the proof is omitted. Denoting by $\lambda_{\mathrm{L}}$ the positive multiplier of Eq. (A.19) and optimizing the corresponding Lagrangean with respect to $\bar{p}, \hat{p}$ and $\underline{p}$ within $[0,1]$ yields:

$$
\begin{aligned}
& \bar{p}_{\mathrm{L}}=1 \Leftrightarrow 2\left(1+\lambda_{\mathrm{L}}\right) q^{2} \underset{\theta}{E}(\tilde{S}(\theta, \tilde{x}(\theta, \bar{\alpha}) \bar{\alpha}))>0, \\
& \hat{p}_{\mathrm{L}} \in[0,1] \Leftrightarrow 2\left(1+\lambda_{\mathrm{L}}\right) q(1-q)(\underset{\theta}{E}(\tilde{S}(\theta, \tilde{x}(\theta, \bar{\alpha}), \bar{\alpha}))+\underset{\theta}{E}(\tilde{S}(\theta, \tilde{x}(\theta, \underline{\alpha}), \underline{\alpha}))) \\
&=2 \lambda_{\mathrm{L}} q^{2}(\underset{\theta}{E}(\tilde{S}(\theta, \tilde{x}(\theta, \bar{\alpha}), \bar{\alpha}))-\underset{\theta}{E}(\tilde{S}(\theta, \tilde{x}(\theta, \underline{\alpha}), \underline{\alpha}))), \\
& \underline{p}_{\mathrm{L}}=0 \Leftrightarrow 2\left(1+\lambda_{\mathrm{L}}\right)(1-q)^{2} \underset{\theta}{E}(\tilde{S}(\theta, \tilde{x}(\theta, \underline{\alpha}), \underline{\alpha})) \\
&\left.\quad<2 \lambda_{\mathrm{L}} q(1-q)(\underset{\theta}{E(\tilde{S}}(\theta, \tilde{x}(\theta, \bar{\alpha}), \bar{\alpha}))-\underset{\theta}{E}(\tilde{S}(\theta, \tilde{x}(\theta, \underline{\alpha}), \underline{\alpha}))\right)
\end{aligned}
$$

Of course, $\bar{p}^{*}=1$ and $p^{*}=0$ imply that Eqs. (A.20) and (A.22) both hold. Inserting $\bar{p}_{\mathrm{L}}=1$, $p_{\mathrm{L}}=0$ and Eq. (A.21) into Eq. (A.19) binding yields:

$$
\underset{\theta}{E}(\tilde{S}(\theta, \tilde{x}(\theta, \bar{\alpha}), \bar{\alpha}))=\frac{\hat{p}_{\mathrm{L}}}{1+\lambda_{\mathrm{L}}}(\underset{\theta}{E}(\tilde{S}(\theta, \tilde{x}(\theta, \bar{\alpha}), \bar{\alpha}))-\underset{\theta}{E}(\tilde{S}(\theta, \tilde{x}(\theta, \underline{\alpha}), \underline{\alpha}))),
$$

and thus $\hat{p}_{\mathrm{L}}>0$.

Using Eq. (A.19) binding, we can derive $\hat{p}_{\mathrm{L}}$ explicitly as:

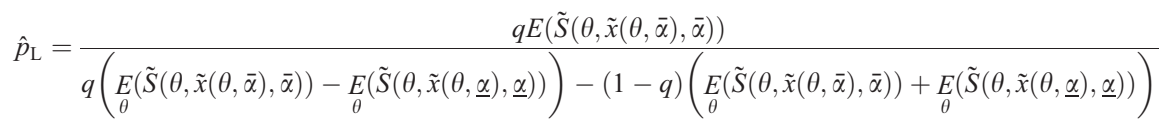

Finally $\hat{p}_{\mathrm{L}}<1$ when condition (25) holds.

\section{Appendix G. Proof of Proposition 6}

We derive from the Proof of Proposition 4 (Eq. (A.15)) that $\lambda$ is given by the following expression:

$$
\frac{\lambda}{1+\lambda}=\frac{(1-q)\left(\underset{\theta}{E}(\tilde{S}(\theta, \tilde{x}(\theta, \bar{\alpha}), \bar{\alpha}))+\underset{\theta}{E}\left(\tilde{S}\left(\theta, x^{\mathrm{SB}}(\theta, \underline{\alpha}, \bar{\alpha}), \underline{\alpha}\right)\right)\right)}{q v \Delta \alpha \Delta \theta v\left(x^{\mathrm{SB}}(\underline{\theta}, \underline{\alpha}, \bar{\alpha})\right)} .
$$


For $\Delta \theta$ small enough, $x^{\mathrm{SB}}(\underline{\theta}, \underline{\alpha}, \bar{\alpha})$ differs from $\tilde{x}(\underline{\theta}, \underline{\alpha})$ by a term of order $\Delta \theta$. Since $\tilde{x}(\underline{\theta}, \underline{\alpha})$ maximizes $\tilde{S}(\underline{\theta}, x, \underline{\alpha})$, the factor in the numerator above differs of $E(\tilde{S}(\theta, \tilde{x}(\theta, \bar{\alpha}), \bar{\alpha}))+E$ $(\tilde{S}(\theta, \tilde{x}(\theta, \underline{\alpha}), \underline{\alpha}))$ by terms of order $\Delta \theta^{2}$. Therefore, we have up to terms of order $\Delta \theta^{2}$ on the right-hand side below

$$
\frac{\lambda \Delta \theta}{1+\lambda}=\frac{(1-q)(\underset{\theta}{E}(\tilde{S}(\theta, \tilde{x}(\theta, \bar{\alpha}), \bar{\alpha}))+\underset{\theta}{E}(\tilde{S}(\theta, \tilde{x}(\theta, \underline{\alpha}), \underline{\alpha})))}{q v \Delta \alpha v(\tilde{x}(\underline{\theta}, \underline{\alpha}))}\left(1-\frac{v^{\prime}(\tilde{x}(\underline{\theta}, \underline{\alpha})) \Delta x}{v(\tilde{x}(\underline{\theta}, \underline{\alpha}))}\right)
$$

where $\Delta x=x^{\mathrm{SB}}(\underline{\theta}, \underline{\alpha}, \bar{\alpha})-\tilde{x}(\underline{\theta}, \underline{\alpha})<0$.

Similarly, we get from the Proof of Proposition 5 that the multiplier $\lambda_{\mathrm{L}}$ is given by

$$
\frac{\lambda_{\mathrm{L}}}{1+\lambda_{\mathrm{L}}}=\frac{(1-q)\left(E_{\theta}(\tilde{S}(\theta, \tilde{x}(\theta, \bar{\alpha}), \bar{\alpha}))+\underset{\theta}{E}(\tilde{S}(\theta, \tilde{x}(\theta, \underline{\alpha}), \underline{\alpha}))\right)}{q\left(E_{\theta}(\tilde{S}(\theta, \tilde{x}(\theta, \bar{\alpha}), \bar{\alpha}))-E_{\theta}(\tilde{S}(\theta, \tilde{x}(\theta, \underline{\alpha}), \underline{\alpha}))\right)} .
$$

We thus observe that:

$$
\frac{\lambda}{1+\lambda} \geq \frac{\lambda_{\mathrm{L}}}{1+\lambda_{\mathrm{L}}}\left(\frac{\underset{\theta}{E(\tilde{S}(\theta, \tilde{x}(\theta, \bar{\alpha}), \bar{\alpha}))-\underset{\theta}{E}(\tilde{S}(\theta, \tilde{x}(\theta, \underline{\alpha}), \underline{\alpha}))}}{v \Delta \alpha \Delta \theta v(\tilde{x}(\underline{\theta}, \underline{\alpha})}\right) .
$$

The bracketed term in the right-hand side above is greater than one since we have

$$
\begin{gathered}
\underset{\theta}{E}(\tilde{S}(\theta, \tilde{x}(\theta, \bar{\alpha}), \bar{\alpha}))-\underset{\theta}{E}(\tilde{S}(\theta, \tilde{x}(\theta, \underline{\alpha}), \underline{\alpha}))-v \Delta \alpha \Delta \theta v(\tilde{x}(\underline{\theta}, \underline{\alpha})) \\
\quad=(1-v)(\tilde{S}(\underline{\theta}, \tilde{x}(\underline{\theta}, \bar{\alpha}), \bar{\alpha}))-\tilde{S}(\underline{\theta}, \tilde{x}(\underline{\theta}, \underline{\alpha}), \bar{\alpha}))>0
\end{gathered}
$$

by definition of $\tilde{x}(\underline{\theta}, \bar{\alpha})$. Finally, we get that $\lambda>\lambda_{\mathrm{L}}$.

Let us turn to the expressions of $\hat{p}^{\mathrm{SB}}$ and $\hat{p}_{\mathrm{L}}$. We have the following approximations (up to terms of order $\Delta \theta^{2}$ )

$$
\hat{p}^{\mathrm{SB}}=\frac{(1+\lambda) \underset{\theta}{E}(\tilde{S}(\theta, \tilde{x}(\theta, \bar{\alpha}), \bar{\alpha}))}{v \Delta \theta \Delta \alpha v(\tilde{x}(\underline{\theta}, \underline{\alpha}))},
$$

and

$$
\hat{p}_{\mathrm{L}}=\frac{\left(1+\lambda_{\mathrm{L}}\right) \underset{\theta}{E}(\tilde{S}(\theta, \tilde{x}(\theta, \bar{\theta}), \bar{\theta}))}{(\underset{\theta}{E(\tilde{S}}(\theta, \tilde{x}(\theta, \bar{\alpha}), \bar{\alpha}))-\underset{\theta}{E}(\tilde{S}(\theta, \tilde{x}(\theta, \underline{\alpha}), \underline{\alpha})))} .
$$

Since $\lambda_{\mathrm{L}}<\lambda$ and $\underset{\theta}{E}(\tilde{S}(\theta, \tilde{x}(\theta, \bar{\alpha}), \bar{\alpha}))-\underset{\theta}{E}(\tilde{S}(\theta, \tilde{x}(\theta, \underline{\alpha}), \underline{\alpha}))>v \Delta \theta \Delta \alpha v(\tilde{x}(\underline{\theta}, \underline{\alpha})), \quad$ we get $\hat{p}_{\mathrm{L}}<\hat{p}^{\mathrm{SB}}$. 


\section{Appendix H. Proof of Proposition 7}

The consolidation of incentive, budget-balanced and participation constraints into Eq. (18) is the same as before and is thus omitted. When $\beta<1$, the $\bar{\alpha}$-country incentive constraint is binding. This leads to the maximand of $(\mathrm{TP})_{\mathrm{RC}} *$. Optimizing the Lagrangean and proceeding exactly as in the Proof of Proposition 4 yields the consumption distortions in the text, whereas probabilities of building the project are given by:

$$
\bar{p}_{\mathrm{RC}}=1 \Leftrightarrow 2(1+\lambda) q^{2} \underset{\theta}{E}(\tilde{S}(\theta, \tilde{x}(\theta, \bar{\alpha}), \bar{\alpha}))>0,
$$

which holds from (H1).

$$
\begin{aligned}
\hat{p}_{\mathrm{RC}} & \in[0,1] \Leftrightarrow 2(1+\lambda) q(1-q)\left(\underset{\theta}{E}(\tilde{S}(\theta, \tilde{x}(\theta, \bar{\alpha}), \bar{\alpha}))+\underset{\theta}{E}\left(\tilde{S}\left(\theta, x_{\mathrm{RC}}(\theta, \underline{\alpha}, \bar{\alpha}), \underline{\alpha}\right)\right)\right) \\
& =2(\lambda+1-\beta) q^{2} v \Delta \alpha \Delta \theta v\left(x_{\mathrm{RC}}(\theta, \underline{\alpha}, \bar{\alpha})\right), \\
\underline{p}_{\mathrm{RC}} & =0 \Leftrightarrow 2(1+\lambda)(1-q)^{2} \underset{\theta}{E}\left(\tilde{S}\left(\theta, x_{\mathrm{RC}}(\theta, \underline{\alpha}, \underline{\alpha}), \underline{\alpha}\right)\right) \\
& <2(\lambda+1-\beta) q(1-q) v \Delta \alpha \Delta \theta v\left(x_{\mathrm{RC}}(\underline{\theta}, \underline{\alpha}, \underline{\alpha})\right) .
\end{aligned}
$$

This latter inequality holds from condition (H2).. From Eq. (18) binding, and taking also into account that $\bar{p}_{\mathrm{RC}}=1$ and $p_{\mathrm{RC}}=0$, we get that $\hat{p}^{\mathrm{SB}}$, if it belongs to $[0,1]$, is the solution to the following equation:

$$
\begin{aligned}
& \hat{p}_{\mathrm{RC}}(1-q)\left(\underset{\theta}{E}(\tilde{S}(\theta, \tilde{x}(\theta, \bar{\alpha}), \bar{\alpha}))+\underset{\theta}{E}\left(\tilde{S}\left(\theta, x_{\mathrm{RC}}(\theta, \underline{\alpha}, \bar{\alpha}), \underline{\alpha}\right)\right)\right)+\underset{\theta}{E}(\tilde{S}(\theta, \tilde{x}(\theta, \bar{\alpha}), \bar{\alpha})) \\
& \quad=q v \hat{p}_{\mathrm{RC}} \Delta \alpha \Delta \theta v\left(x_{\mathrm{RC}}(\underline{\alpha}, \bar{\alpha})\right) .
\end{aligned}
$$

Using Eqs. (A.25) and (A.27), we obtain:

$$
\hat{p}_{\mathrm{RC}} \frac{v \beta}{1+\lambda} \Delta \alpha \Delta \theta v\left(x_{\mathrm{RC}}(\underline{\theta}, \underline{\alpha}, \bar{\alpha})\right)=\underset{\theta}{E}(\tilde{S}(\theta, \tilde{x}(\theta, \bar{\alpha}), \bar{\alpha})) .
$$

The right-hand side is positive by condition (H1). Hence, $\hat{p}_{\mathrm{RC}}>0$. As in the Proof of Proposition 4 , one can show that $\hat{p}_{\mathrm{RC}}<1$ is necessary when condition (19) holds.

\section{Appendix I. Proof of Proposition 8}

The consolidation of incentive, budget-balanced and participation constraints into Eq. (18) is the same as before and is thus omitted. When Eq. (19) holds, the $\bar{\alpha}$-country incentive constraint is binding. This leads to the maximand of $(\mathrm{TP})_{\mathrm{RA}}{ }^{*}$. Optimizing the Lagrangean and proceeding exactly as in the Proof of Proposition 4 with the new conditions $\left(\mathrm{H}^{\prime}\right)$ and $\left(\mathrm{H} 2^{\prime}\right)$ yields the probabilities and consumptions in the text. 


\section{References}

Arce, D., Sandler, T., 2002. Transnational public goods; strategies and institutions. Working Paper. University of Southern California.

Arrow, K., 1979. The property rights doctrine and demand revelation under incomplete information. Economics and Human Welfare. Academic Press.

Calderon, C., Serven, L., 2002. The output cost of Latin America's infrastructure lag. In: Calderon, C., Easterly, W., Serven, L. (Eds.), The Macroeconomics of Infrastructure in Latin America.

Calderon, C., Easterly, W., Serven, L. (Eds.), 2002. The Macroeconomics of Infrastructure in Latin America.

D’Aspremont, C., Grard-Varet, L.A., 1979. Incentives and incomplete information. Journal of Public Economics $11,25-45$.

Faure-Grimaud, A., Laffont, J.J., Martimort, D., 2003. Collusion and supervision with soft information. Review of Economic Studies 70, 253-280.

Green, J., Laffont, J.J., 1977a. Revelation des Préférénces pour les Biens Publics. Cahiers du Séminaire D’ Économetrie 19, 83-103.

Green, J., Laffont, J.J., 1977b. Characterization of satisfactory mechanisms for the revelation of preferences for public goods. Econometrica 45, 427-438.

Groves, T., 1973. Incentives in teams. Econometrica 41, 617-631.

Guasch, L., Laffont, J.J., Straub, S., 2002. Renegotiation of Concession Contracts in Latin America, mimeo IDEI.

Holmström, B., Myerson, R., 1983. Efficient and durable decision rules with incomplete information. Econometrica $51,1799-1819$.

Laffont, J.J., Martimort, D., 2002. The Theory of Incentives: The Principal-Agent Model. Princeton Univ. Press, Princeton.

Laffont, J.J., Maskin, E., 1979. A differential approach to expected utility maximizing mechanisms. In: Laffont, J.J. (Ed.), Aggregation and Revelation of Preferences. North-Holland, Amsterdam, pp. $289-308$.

Laussel, D., Palfrey, T., 2003. Efficient equilibria in the voluntary contributions mechanisms with private information. Journal of Public Economic Theory 5, 449-478.

Ledyard, J., Palfrey, T., 1999. A characterization of interim efficiency with public goods. Econometrica 64, 435-448.

Mailath, G., Postlewaite, A., 1990. Asymmetric information bargaining problems with many agents. Review of Economic Studies 57, 351-367.

Makowski, L., Mezzetti, C., 1994. Bayesian and weakly robust first-best mechanisms: characterization. Journal of Economic Theory 64, 500-519.

Martimort, D., 2001. Optimal taxation and strategic budget deficit under political regime switching. Review of Economic Studies 68, 573-592.

Martimort, D., Moreira, H., 2003. Common agency with informed principals, mimeo Toulouse.

Maskin, E., 1999. Nash equilibrium and welfare optimality. Review of Economic Studies 66, 23-38.

Maskin, E., Tirole, J., 1990. The principal-agent relationship with an informed principal I: private-values. Econometrica 58, 379-410.

McAfee, P., McMillan, J., 1995. Organizational diseconomies of scope. Journal of Economics and Management Strategy 4, 399-426.

Melumad, N., Mookherjee, D., Reichelstein, S., 1995. Hierarchical decentralization of incentive contracts. Rand Journal of Economics 26, 654-672.

Menezes, F., Monteiro, P., Temini, A., 2001. Private provision of discrete public goods with incomplete information. Journal of Mathematical Economics 35, 493-514.

Mookherjee, D., Reichelstein, S., 1992. Dominant strategy implementation of Bayesian incentive compatible allocation rules. Journal of Economic Theory 56, 378-399.

Myerson, R., Sattherwaite, M., 1983. Efficient mechanisms for bilateral trading. Journal of Economic Theory 28, 265-281.

Rob, R., 1989. Pollution claims settlements with private information. Journal of Economic Theory 47, $307-333$.

Sandler, T., 1998. Global and regional public goods: a prognosis for collective action. Fiscal Studies 19, $221-247$.

Sandler, T., 2001. On financing global and international public goods. In: Mody, A. (Ed.), International Public Goods: Incentives, Measurements and Financing. Kluwer Academic Publishing, Dordecht, NL, pp. 221 - 247. 
Schiff, M., Winters, A., 2002. Regional cooperation, and the role of international organizations and regional integration. World Bank Policy Research Working Paper 2872. Washington.

Walker, M., 1980. On the inexistence of a dominant strategy mechanism for making optimal public good decisions. Econometrica 48, 1521-1540.

Williams, S., 1999. A characterization of efficient Bayesian incentive compatible mechanism. Economic Theory $14,155-180$.

World Development Report, 1994. Infrastructure for Development. World Bank, Oxford Univ. Press. 